\title{
Momentum broadening of energetic partons in an anisotropic plasma
}

\author{
Sigtryggur Hauksson $\odot,{ }^{*}$ Sangyong Jeon, and Charles Gale \\ Department of Physics, McGill University, 3600 University Street, Montréal, Quebec, Canada H3A 2T8
}

(Received 7 October 2021; accepted 12 January 2022; published 27 January 2022)

\begin{abstract}
The quark-gluon plasma produced in heavy-ion collisions is anisotropic throughout its evolution. This anisotropy changes the physics of jet-medium interaction, making it dependent on the momentum direction of the jet. In this paper we analyze transverse momentum broadening of a jet parton interacting with soft gluons in an anisotropic plasma. Our analysis equally applies to momentum broadening of quasiparticles in kinetic theory. We subtract contribution from instability modes in the deep infrared and discuss how our calculation should be complemented in that regime. The resulting anisotropic collision kernel for momentum broadening is qualitatively different from the equilibrium collision kernel and from the isotropic ansatz used in effective kinetic theory. Because of increased medium screening, there is substantially less transverse broadening at low and intermediate momenta.
\end{abstract}

DOI: 10.1103/PhysRevC.105.014914

\section{INTRODUCTION}

Heavy-ion collisions at RHIC and the LHC produce extremely dense and energetic matter governed by the strong interaction: this matter is known as the quark-gluon plasma (QGP) [1]. One of the primary experimental probes of the QGP are jets which are produced during the initial hard scattering of nuclei. As these jets traverse the droplet of QGP their structure is modified, leaving imprints of the medium on experimental observables.

A great deal of theoretical effort has explored how nearly on-shell jet partons interact with a weakly coupled QGP medium. A jet parton receives repeated small momentum kicks from medium particles leading to diffusion in the parton's momentum transverse to its direction of motion. These kicks bring the jet parton slightly off shell, allowing it to radiate gluons through medium-induced branching, which changes the jet shower relative to vacuum. In addition, jets lose energy when traversing the plasma as they interact with their radiation field, and low-energy jet partons are absorbed by the medium.

Using thermal field theory, one can evaluate transverse momentum broadening in a weakly coupled plasma. This is quantified by the collision kernel $\mathcal{C}\left(\mathbf{q}_{\perp}\right)$ which is the probability of receiving a transverse kick of momentum $\mathbf{q}_{\perp}$ from the medium. Such microscopic calculations exist at leading order in perturbation theory [2] as well as at next-to-leading order [3]. The collision kernel for transverse momentum

*Corresponding author: sigtryggur.hauksson@mail.mcgill.ca

Published by the American Physical Society under the terms of the Creative Commons Attribution 4.0 International license. Further distribution of this work must maintain attribution to the author $(s)$ and the published article's title, journal citation, and DOI. Funded by $S C O A P^{3}$. broadening has furthermore been evaluated nonperturbatively on the lattice using electrostatic QCD effective field theory (see, e.g., [4,5]).

Analytic calculations of the collision kernel have so far assumed a medium in local thermal equilibrium, or with local isotropy in momentum space [6]. However, we know that there are sizable deviations from isotropy and thermal equilibrium at all stages of heavy-ion collisions [7]. This can have important effects on the phenomenology of jets: an anisotropy in the local momentum distribution of quarks and gluons leads to directional dependence in jet evolution. Specifically, jet partons going through the same patch of QGP but traveling in different directions will have different rates of momentum broadening and of medium-induced splitting.

Having an anisotropic collision kernel for momentum broadening is not only important for jet physics, but also for formulating a kinetic theory of quarks and gluons [8]. Such kinetic theories are used to describe early stages of heavy-ion collisions after the glasma phase and before the hydrodynamic phase $[9,10]$. One of the two main processes for quasiparticle interaction is gluon radiation by a quark or a gluon which is brought slightly off-shell by momentum broadening. Up until now, kinetic theory calculations have employed an isotropic ansatz for the collision kernel [11,12] but consistency requires a nonequilibrium kernel. This could affect results of kinetic theory simulations. We finally note that calculation of photon radiation through bremsstrahlung in an anisotropic medium relies on precisely the same nonequilibrium collision kernel $[6,13,14]$.

Understanding momentum broadening in an anisotropic plasma requires a detailed microscopic calculation. In this paper we provide such a calculation in the hard thermal loop (HTL) regime where self-interaction of soft gluons can be ignored. Specifically, we consider a medium in which quark and gluon quasiparticles are distributed anisotropically in momentum space. The quasiparticles source soft gluons which propagate until they give the jet parton a transverse kick. This 
gives rise to a collision kernel $\mathcal{C}\left(\mathbf{q}_{\perp}\right)$ which depends not only on local properties of the medium but also on the direction of the jet parton. Our calculation is at leading order in perturbation theory. We subtract instability modes [15] corresponding to exponential growth in soft gluon density [16], as they have become saturated during the kinetic and hydrodynamic stages we are interested in [17-19].

We note that numerical simulations have measured momentum broadening of jet partons and heavy quarks in a variety of equilibrium and nonequilibrium situations. These include classical-statistical field theory [20], an HTL setup with kinetic theory for quasiparticles and classical field theory for soft gluons [21-23], as well as the color-glass condensate (see [24-26]). Furthermore, momentum broadening has been measured on the lattice in a factorized approach assuming a single scattering off medium gluons [27]. The effect of instabilities on momentum broadening was furthermore assessed in $[28,29]$. We also note that [30] evaluated the effect of temperature and density gradients on momentum broadening and jet splitting, assuming a medium composed of massive particles and working in an opacity expansion. Our analytic approach complements these studies as it gives results which are independent of assumptions of numerical simulations. Furthermore, our results only depend on the instantaneous properties of the medium. We evaluate the full collision kernel which is needed for the rate of gluon radiation.

The paper is organized as follows: In Sec. II we discuss the physics of jet momentum broadening in detail and show how it differs microscopically from jet energy loss, in a nonequilibrium medium. In Sec. III we calculate the density of soft gluons in an anisotropic medium. In Sec. IV we discuss our treatment of instabilities in an anisotropic plasma. Finally, results are presented in Sec. V. Some additional details are provided in the Appendices.

\section{JET MOMENTUM BROADENING AND ENERGY LOSS}

As a jet parton passes through a plasma it loses energy and gains momentum transverse to its direction of motion. This happens through three different processes: hard twoto-two scattering with plasma constituents, medium-induced bremsstrahlung, and interaction with soft gluons [31]. In this paper we focus on interaction with soft gluons, which has been less studied in a nonequilibrium medium and which furthermore is the basis of medium-induced splitting.

In thermal equilibrium the physics of energy loss due to soft gluons is different from that of momentum broadening from soft gluons: momentum broadening results from transverse kicks of gluons that exist in the medium and have been radiated by quasiparticles, while energy loss results from soft gluons that the jet parton itself radiates, and not gluons present in the medium.

It is important to establish that energy loss and momentum broadening differ in the same way in a nonequilibrium system, i.e., that momentum broadening is due to soft gluons in the medium while energy loss is due to the soft gluon radiation field of the parton itself. We will show this using the realtime formalism $[32,33]$ which will furthermore establish our conventions. We focus on an energetic quark parton traversing

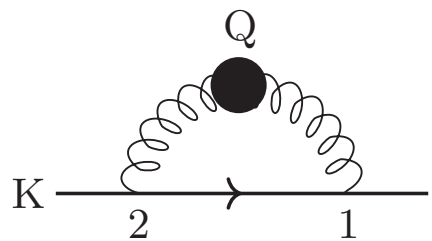

FIG. 1. Self-energy diagram for a jet quark $K$ interacting with a soft gluon $Q$. The soft gluon propagator is resummed.

the plasma but the argument for a gluon parton or a heavy quark is nearly identical.

A quark parton flying through the plasma with momentum $K^{\mu}=\left(k^{0}, \mathbf{k}\right)$ interacts with soft gluons. This leads to a decay rate [32]

$$
\Gamma=\frac{1}{4 k} \operatorname{Tr}\left[\not K \Sigma_{21}\right]
$$

where the self-energy component

$$
\begin{aligned}
\Sigma_{21}(K)= & g^{2} C_{F} \int \frac{d^{4} Q}{(2 \pi)^{4}} D_{21}^{\mu \nu}(Q) \gamma_{\mu}(\mathbb{K}-\mathscr{Q}) \\
& \times \gamma_{\nu}\left[1-f_{q}(\mathbf{k}-\mathbf{q})\right] 2 \pi \delta\left[(K-Q)^{2}\right]
\end{aligned}
$$

is given by the diagram in Fig. 1. Using that the soft gluon momentum $Q$ is much smaller than the jet parton momentum $K$ we get a differential decay rate

$$
\frac{d \Gamma}{d^{4} Q}=\frac{g^{2} C_{F}}{(2 \pi)^{4}} D_{21}^{\mu \nu}(Q) v_{\mu} v_{\nu} \delta(v \cdot Q)
$$

where $v_{\mu}=K^{\mu} / k$ is the normalized parton momentum. To generalize our results for a heavy quark, one simply substitutes $v^{\mu}$ with $\left(k^{0} / k, \widehat{\mathbf{k}}\right)=\left(\sqrt{k^{2}+m^{2}} / k, \widehat{\mathbf{k}}\right)$. In either case, the essential ingredient is the resummed propagator for soft gluons

$$
D_{21}^{\mu \nu}(Q)=\int d^{4}(x-y) e^{i Q(x-y)}\left\langle A^{\mu}(x) A^{v}(y)\right\rangle
$$

which can be written more physically as ${ }^{1}$

$$
D_{21}^{\mu \nu}(Q)=\frac{1}{2}\left[D_{\text {ret }}^{\mu \nu}(Q)-D_{\text {adv }}^{\mu \nu}(Q)\right]+D_{r r}^{\mu \nu}(Q)
$$

The two terms in Eq. (5) have different interpretations. The $r r$ propagator is

$$
D_{r r}^{\mu \nu}(x, y)=\frac{1}{4}\left\langle\left\{A^{\mu}(x), A^{\nu}(y)\right\}\right\rangle .
$$

It is nonvanishing even for classical fields, and describes the density of soft gluons in the medium. The contribution of the $r r$ correlator to the decay rate in Eq. (3) thus corresponds to the jet parton interacting with soft gluons that are already present in the medium.

\footnotetext{
${ }^{1}$ In a nonequilibrium plasma one uses a Wigner transform $D_{21}^{\mu \nu}(Q, X)=\int d^{4}(x-y) e^{i Q(x-y)}\left\langle A^{\mu}(x) A^{v}(y)\right\rangle$ where $X=$ $(x+y) / 2$ is the position in the plasma. In this section we assume that changes in $X$ are slow and we omit writing dependence on $X$ explicitly.
} 
Meanwhile, the retarded correlator in Eq. (5) is given by

$$
D_{\text {ret }}^{\mu v}(x, y)=\theta\left(t_{x}-t_{y}\right)\left\langle\left[A^{\mu}(x), A^{v}(y)\right]\right\rangle .
$$

It describes the causal propagation of soft gluons forward in time. Similarly, the advanced correlator is $D_{\text {adv }}(x, y)=$ $-\theta\left(t_{y}-t_{x}\right)\left\langle\left[A^{\mu}(x), A^{v}(y)\right]\right\rangle$. The contribution of these correlators to the differential decay rate describes soft gluons radiated by the jet parton. These soft gluons propagate in the medium before giving the same parton a kick. In other words, in the regime of soft gluons the contribution of the retarded and advanced correlators does not represent gluons already present in the medium, but rather the interaction of the jet with its own radiation field.

The rate of energy loss of a jet parton due to soft gluons is

$$
\widehat{e}=\frac{d\left\langle p^{0}\right\rangle}{d t}=\int d^{4} Q q^{0} \frac{d \Gamma}{d^{4} Q} .
$$

Since $D_{r r}^{\mu \nu}(x, y)=D_{r r}^{v \mu}(y, x)$, one gets that

$$
D_{r r}^{\mu \nu}(Q) v_{\mu} v_{\nu}=D_{r r}^{\mu \nu}(-Q) v_{\mu} v_{\nu} .
$$

Thus, the jet is equally likely to gain four-momentum $Q$ from soft gluons in the medium as to lose four-momentum $Q$. Therefore,

$$
\widehat{e}=g^{2} C_{F} \int \frac{d^{4} Q}{(2 \pi)^{4}} q^{0} \frac{1}{2}\left(D_{\mathrm{ret}}^{\mu v}-D_{\mathrm{adv}}^{\mu v}\right)(Q) v_{\mu} v_{v} 2 \pi \delta(v Q),
$$

which is solely due to the jet's radiation field.

Equation (10) for energy loss has a simple physical interpretation given, e.g., in [34,35]. The jet parton's radiation field is described by classical field theory. The rate of energy loss is then

$$
\widehat{e}=\operatorname{Re} \int d^{3} x \mathbf{J}_{\text {ext }}(x) \cdot \mathbf{E}_{\text {ind }}(x)
$$

where the current is the jet parton itself, $\mathbf{J}_{\mathrm{ext}}=g \mathbf{v} \delta^{(3)}(\mathbf{x}-\mathbf{v} t)$, and $\mathbf{E}_{\text {ind }}$ is the electric field it induces. Linear response theory gives that

$$
E_{\text {ind }}^{i}(Q)=i q^{0} D_{\text {ret }}^{i j}(Q) J_{\text {ext }}^{j}(Q) .
$$

Combining these equations and taking care of color factors reproduces our formula for energy loss, Eq. (10). This shows that energy loss is indeed due to fields induced by the jet parton. ${ }^{2}$

Equation (10) for energy loss has been evaluated in an anisotropic medium along with the contribution of hard medium particles [37,38] (see Appendix D for a discussion). Their formalism was based on the equilibrium calculation in $[39,40]$. Jet energy loss has furthermore been evaluated in an evolving unstable plasma [36] (see also [22]).

\footnotetext{
${ }^{2}$ The expression given in $[34,35]$ has $D_{\text {ret }}-D_{\text {ret }}^{0}$ instead of $D_{\text {ret }}$ where $D_{\text {ret }}^{0}$ is the bare propagator. It is easy to see that the contribution of the bare propagator is phase space suppressed and can be omitted. Their expression furthermore assumes a temporal axial gauge, $A^{0}=0$. In a more general gauge, current conservation needs to be postulated explicitly for the classical argument to go through (see [36]).
}

The physics of jet momentum broadening differs from that of energy loss. In the HTL regime $D_{r r}(Q) \sim 1 / g^{3}$ because of high occupation density of soft gluons while $D_{\text {ret }} \sim 1 / g^{2}$. This can be seen immediately in thermal equilibrium from Eq. (17), and in an anisotropic system we will show this below. The rate of jet transverse momentum broadening is therefore

$$
\begin{aligned}
\widehat{q} & =\frac{d\left\langle\left(\Delta p_{\perp}\right)^{2}\right\rangle}{d t}=\int d^{4} Q q_{\perp}^{2} \frac{d \Gamma}{d^{4} Q} \\
& \approx \int \frac{d^{2} q_{\perp}}{(2 \pi)^{2}} q_{\perp}^{2} \mathcal{C}\left(\mathbf{q}_{\perp}\right)
\end{aligned}
$$

where the collision kernel for momentum broadening is

$$
\mathcal{C}\left(\mathbf{q}_{\perp}\right)=g^{2} C_{F} \int \frac{d q^{0} d q^{z}}{(2 \pi)^{2}} D_{r r}^{\mu \nu}(Q) v_{\mu} v_{\nu} 2 \pi \delta(v \cdot Q)
$$

with $z$ the direction of motion of the jet. We see that jet momentum broadening is due to soft gluons already present in the medium and not due to the jet's radiation field. The main task of this paper is to calculate the collision kernel $\mathcal{C}\left(\mathbf{q}_{\perp}\right)$ and the transport coefficient $\widehat{q}$ in an anisotropic plasma. ${ }^{3}$ This requires evaluating the $r r$ correlator microscopically in a nonequilibrium plasma. We note that longitudinal momentum broadening is

$$
\widehat{q}_{L}:=\frac{d\left\langle\left(\Delta p_{z}\right)^{2}\right\rangle}{d t}=\int d^{4} Q q_{z}^{2} \frac{d \Gamma}{d^{4} Q}
$$

for a parton traveling in the $z$ direction which can similarly be shown to be

$$
\widehat{q}_{L} \approx g^{2} C_{F} \int \frac{d^{4} Q}{(2 \pi)^{4}} q_{z}^{2} D_{r r}^{\mu \nu}(Q) v_{\mu} v_{\nu} 2 \pi \delta(v \cdot Q) .
$$

In thermal equilibrium, many of our results simplify. The Kubo-Martin-Schwinger (KMS) relation gives that

$$
D_{r r}^{\mu \nu}(Q)=\left(\frac{1}{2}+f_{\mathrm{B}}\left(q^{0}\right)\right)\left[D_{\mathrm{ret}}^{\mu v}(Q)-D_{\mathrm{adv}}^{\mu v}(Q)\right],
$$

where $f_{\mathrm{B}}\left(q^{0}\right)=1 /\left(e^{q^{0} / T}-1\right)$ is the Bose-Einstein distribution. Using that $f_{\mathrm{B}}\left(q^{0}\right) \approx T / q^{0}$ for soft gluons, the KMS relation leads to

$$
\widehat{q}_{L}=T \widehat{e}
$$

which is a fluctuation-dissipation relation linking momentum broadening due to fluctuating soft gluons in the medium and energy loss which happens through dissipation by the jet's radiation field. Furthermore, in thermal equilibrium, the integral in Eq. (14) can be evaluated giving a simple, analytic result for the collision kernel $[2,3]$

$$
\mathcal{C}\left(\mathbf{q}_{\perp}\right)=g^{2} C_{F} T\left(\frac{1}{\mathbf{q}_{\perp}^{2}}-\frac{1}{\mathbf{q}_{\perp}^{2}+m_{D}^{2}}\right)
$$

\footnotetext{
${ }^{3}$ References [41,42] aimed to evalute momentum broadening in an anisotropic plasma. We believe they incorrectly assumed a KMS condition in the nonequilibirum setup [see, e.g., Eq. (5) in [41]]. Doing so ignores the details of how soft gluons are emitted by an anisotropic distribution of quasiparticles.
} 
where $T$ is the temperature and $m_{D}^{2}$ is the Debye mass. We emphasize that Eqs. (17)-(19) do not hold out of equilibrium and the $r r$ propagator and the collision kernel $\mathcal{C}$ need to be evaluated in detail.

\section{DENSITY OF SOFT GLUONS IN AN ANISOTROPIC MEDIUM}

The collision kernel in Eq. (14) for transverse momentum broadening depends on soft gluon density through the $r r$ correlator. We will now derive the correlator's symmetric component in an anisotropic medium. The state of the medium is determined by the momentum distribution of hard quarks and gluons which are distributed anisotropically in momentum space. We assume that an equilibrium distribution, i.e., a Bose-Einstein distribution for gluons and a Fermi-Dirac distribution for quarks, is elongated or contracted in a direction n. This leads to the momentum distribution introduced by Romatschke and Strickland [43],

$$
f(\mathbf{p})=\sqrt{1+\xi} f_{\text {eq }}\left(\sqrt{p^{2}+\xi(\mathbf{n} \cdot \mathbf{p})^{2}}\right),
$$

where the parameter $\xi$ quantifies the degree of anisotropy. We use $\Lambda$ for the parameter corresponding to temperature in thermal equilibrium. The normalization factor $\sqrt{1+\xi}$ guarantees that the number density of hard particles is the same as in thermal equilibrium. More general anisotropic distributions and the corresponding plasmons have been considered in the literature (see, e.g., [44,45]). These would lead to an even more involved calculation of the $r r$ correlator.

In momentum space the $r r$ correlator is

$$
D_{r r}^{\mu \nu}(Q)=D_{\text {ret }}^{\mu \omega}(Q) \Pi_{a a}^{\omega \xi}(Q) D_{\mathrm{adv}}^{\xi v}(Q),
$$

where identical indices are contracted. ${ }^{4}$ Here two soft gluon excitations are sourced with probability $\epsilon_{\mu} \epsilon_{\nu}^{*} \Pi_{a a}^{\mu \nu}$ where $\epsilon_{\mu}$ is the gluon polarization. The index $a a$ on the self-energy comes from the $r / a$ basis in the real-time formalism [33]. The gluons then evolve in time as given by $D_{\text {ret }}$ and $D_{\text {adv }}$. This expression assumes a system initialized at time $t_{0}=-\infty$ with the initial condition specified by quark and gluon momentum distributions. It furthermore assumes that the medium changes slowly enough to use Fourier transforms. We will discuss the validity of these assumption in Sec. IV.

We assume that the system is in the HTL regime. The hard quasiparticles at energy $\Lambda$ then source soft gluons at energy $g \Lambda$. These soft gluons have occupancy of order $\sim 1 / g$ and can be described with classical field theory. Their self-interaction is suppressed relative to interaction with hard quasiparticles [46]. In this regime

$$
\begin{aligned}
\Pi_{a a}^{\mu \nu}= & \left.g^{2} \int \frac{d^{3} p}{(2 \pi)^{3}} v^{\mu} v^{v} 2 \pi \delta(v \cdot Q)\right|_{v=(1, \widehat{\mathbf{p}})} \\
& \times\left\{2 N_{f} f_{q}(\mathbf{p})\left[1-f_{q}(\mathbf{p})\right]+2 N_{c} f_{g}(\mathbf{p})\left[1+f_{g}(\mathbf{p})\right]\right\},
\end{aligned}
$$

\footnotetext{
${ }^{4}$ We use modern summation convention where $A^{\mu} B^{\mu}=A_{\mu} B^{\mu}=$ $g_{\mu \nu} A^{\mu} B^{\nu}$.
}

which gives the radiation of soft gluons by hard quarks, $f_{q}(\mathbf{p})$, and hard gluons, $f_{g}(\mathbf{p})$, including Bose enhancement and Pauli blocking [8]. As usual, $N_{f}$ is the number of quark flavors and $N_{c}$ is the number of colors. The retarded correlator $D_{\text {ret }}^{\mu \nu}$ in the HTL approximation in the Feynman gauge is

$$
D_{\text {ret }}^{\mu \nu}=i\left(\left[P^{2}-\Pi_{\mathrm{ret}}\right]^{-1}\right)^{\mu \nu}
$$

where

$$
\Pi_{\text {ret }}^{\mu \nu}(Q)=-\left.g^{2} \int \frac{d^{3} p}{(2 \pi)^{3}} \frac{\partial f_{\text {tot }}}{\partial P^{\omega}}\left[-v^{\mu} g^{\omega \nu}+\frac{Q^{\omega} v^{\mu} v^{v}}{v \cdot Q+i \epsilon}\right]\right|_{v=(1, \hat{\mathbf{p}})}
$$

with $f_{\text {tot }}(\mathbf{p})=2 N_{f} f_{q}(\mathbf{p})+2 N_{c} f_{g}(\mathbf{p})$ [47]. It describes the propagation of soft gluons as they interact with hard particles in the medium. This retarded correlator has been evaluated in [43] with the momentum distribution in Eq. (20) (see also [48] for a detailed discussion). We will reproduce this derivation for completeness as our conventions differ. The advanced propagator is given by $D_{\mathrm{adv}}=D_{\mathrm{ret}}^{\dagger}$. It is easy to see that $D_{r r} \sim 1 / g^{3}$.

Using the ingredients we have assembled, we can evaluate the $r r$ correlator in an anisotropic plasma defined by the momentum distribution in Eq. (20). This requires handling the tensor indices correctly. There are only four tensors in our medium: the metric $g^{\mu \nu}$, the soft gluon momentum $Q^{\mu}=\left(q^{0}, \mathbf{q}\right)$, the fluid's velocity which is $u^{\mu}=(1, \mathbf{0})$ in the fluid's rest frame, as well as the direction of the anisotropy $n^{\mu}=(0, \mathbf{n})$ which is defined to be a spatial vector. The calculation could be done in a different rest frame which would give identical results. It is convenient to define a new anisotropy vector as $\tilde{n}^{\mu}=\left(0, \frac{\hat{\mathbf{n}}^{i}}{\sqrt{\hat{n}^{2}}}\right)$ where

$$
\hat{\mathbf{n}}=\mathbf{n}-\frac{\mathbf{q} \cdot \mathbf{n}}{\mathbf{q}^{2}} \mathbf{q} \text {. }
$$

This guarantees that $\tilde{n}^{\mu}$ is a unit spatial vector, orthogonal to both $u^{\mu}$ and $Q^{\mu}$.

Using $g^{\mu \nu}, Q^{\mu}, u^{\mu}$, and $\tilde{n}^{\mu}$, we can construct seven symmetric, second-rank tensors. ${ }^{5}$ Additionally, both $\Pi_{a a}$ and $\Pi_{\text {ret }}$ satisfy $Q_{\mu} \Pi^{\mu \nu}=0$ as a result of gauge invariance in the HTL approximation. This requirement gives three independent equations since there are three vectors in $v$. Therefore, the number of tensors we need to express the self-energies is reduced to 4 . These four tensors can be chosen as any linear combination $X^{\mu \nu}$ of the seven original tensors that satisfies $Q_{\mu} X^{\mu \nu}=0$. In the fluid's rest frame, a convenient choice for the first tensor is

$$
P_{T}^{i j}=\delta^{i j}-\frac{q^{i} q^{j}}{\mathbf{q}^{2}}
$$

which is transverse to the gluon momentum, with all other components zero. We choose the second tensor to be longitudinal to the gluon momentum:

$$
P_{L}^{\mu \nu}=\frac{Q^{\mu} Q^{\nu}}{Q^{2}}-g^{\mu \nu}-P_{T}^{\mu \nu} .
$$

\footnotetext{
${ }^{5}$ These can for example be chosen to be $g^{\mu \nu}, Q^{\mu} Q^{v}, u^{\mu} u^{v}, \tilde{n}^{\mu} \tilde{n}^{v}$, $Q^{\mu} u^{\nu}+u^{\mu} Q^{\nu}, Q^{\mu} \tilde{n}^{v}+\tilde{n}^{\mu} Q^{v}$, and $u^{\mu} \tilde{n}^{v}+\tilde{n}^{\mu} u^{\nu}$.
} 
Both of these tensors are present in thermal equilibrium [49]. The third tensor describes propagation along the anisotropy axis:

$$
C^{\mu \nu}=\tilde{n}^{\mu} \tilde{n}^{\nu}
$$

Finally, the fourth tensor is given by

$$
\begin{aligned}
& D^{00}=0, \\
& D^{0 i}=D^{i 0}=\frac{\mathbf{q}^{2}}{q^{0}} \tilde{n}^{i}, \\
& D^{i j}=q^{i} \tilde{n}^{j}+q^{j} \tilde{n}^{i},
\end{aligned}
$$

which mixes the anisotropy direction and the gluon threemomentum. ${ }^{6}$ The most convenient choice of the four tensors turns out to be $P_{L}, C, D$, and $E=P_{T}-C$, which we will use in what follows. An alternative definition of tensors is found in [50].

Using this basis of tensors, the $a a$ self-energy is

$$
-i \Pi_{a a}=\alpha P_{L}+\beta E+\gamma C+\delta D,
$$

and it is straightforward to show that

$$
\begin{aligned}
\alpha & =\frac{Q^{2}}{\mathbf{q}^{2}} \Pi^{00}, \\
\gamma & =\Pi^{i j} \tilde{n}^{i} \tilde{n}^{j}, \\
\delta & =\frac{q^{0}}{\mathbf{q}^{2}} \Pi^{0 i} \tilde{n}^{i}, \\
\beta & =-\alpha-\gamma-\Pi_{\mu}^{\mu} .
\end{aligned}
$$

Explicit expressions for the components are collected in Appendix $\mathrm{C}$, with one integral left to be done numerically. Similarly, the retarded self-energy is

$$
-i \Pi_{\mathrm{ret}}^{\mu \nu}=\Pi_{L} P_{L}^{\mu \nu}+\Pi_{e} E^{\mu \nu}+\Pi_{c} C^{\mu \nu}+\Pi_{d} D^{\mu \nu}
$$

with the components, which reproduce [43], found in Appendix C. Substituting in Eq. (23) then gives the wellknown result for the retarded propagator in the Feynman gauge:

$$
\begin{aligned}
D_{\mathrm{ret}}^{\mu \nu}= & \frac{-i Q^{\mu} Q^{\nu}}{\left(Q^{2}\right)^{2}}+i E^{\mu \nu} \tilde{D}_{\mathrm{ret}}^{B}+i\left[\left(Q^{2}-\Pi_{c}\right) P_{L}^{\mu \nu}\right. \\
& \left.+\left(Q^{2}-\Pi_{L}\right) C^{\mu \nu}+\Pi_{d} D^{\mu \nu}\right] \tilde{D}_{\mathrm{ret}}^{A}
\end{aligned}
$$

where the denominators $\tilde{D}_{\text {ret }}^{A}$ and $\tilde{D}_{\text {ret }}^{B}$ are defined in Eqs. (42) and (43).

In order to derive the $r r$ correlator, we must contract different tensors. This can conveniently be described by commutators and anticommutators. The anticommutator

$$
\{X, Y\}^{\mu \nu}=X^{\mu \omega} Y_{\omega}^{v}+Y^{\mu \omega} X_{\omega}^{v}
$$

\footnotetext{
${ }^{6}$ In a general rest frame this tensor can be written as $D^{\mu \nu}=Q^{\mu} \tilde{n}^{v}+$ $\tilde{n}^{\mu} Q^{v}-\frac{Q^{2}}{u Q}\left(u^{\mu} \tilde{n}^{v}+\tilde{n}^{\mu} u^{v}\right)$, which manifestly obeys $Q_{\mu} D^{\mu v}=0$.
}

is guaranteed to be symmetric so our set of four tensors is closed under anticommutation. In fact, one can show that

$$
\begin{aligned}
P_{L}^{2} & =-P_{L}, \\
E^{2} & =-E, \\
C^{2} & =-C, \\
D^{2} & =-\frac{Q^{2} \mathbf{q}^{2}}{q_{0}^{2}}\left(C+P_{L}\right), \\
\left\{E, P_{L}\right\} & =\{E, C\}=\{E, D\}=\left\{P_{L}, C\right\}=0, \\
\left\{P_{L}, D\right\} & =\{C, D\}=-D .
\end{aligned}
$$

It can be shown that our choice of tensors makes the greatest number of anticommutators vanish, justifying the choice of $E=P_{T}-C$. Nevertheless, the tensors do not form an orthogonal basis as some anticommutators of different tensors do not vanish.

Momentum broadening depends on $D_{r r}^{\mu \nu} \widehat{K}_{\mu} \widehat{K}_{v}$ so we only need the symmetric part:

$$
\begin{aligned}
D_{r r}^{(\mu \nu)} & :=\frac{1}{2}\left[D_{r r}+D_{r r}^{\dagger}\right]^{\mu \nu} \\
& =\frac{1}{2}\left[D_{\mathrm{ret}}\left(-i \Pi_{a a}\right) D_{\mathrm{adv}}+D_{\mathrm{adv}}\left(-i \Pi_{a a}\right) D_{\mathrm{ret}}\right]^{\mu \nu} .
\end{aligned}
$$

Using the general relation

$$
X Y Z+Z Y X=\frac{1}{2}[\{X,\{Y, Z\}\}-\{Y,\{Z, X\}\}+\{Z,\{X, Y\}\}]
$$

for tensors $X, Y, Z$, we can evaluate the symmetric component of $D_{r r}$ using the anticommutation relations in Eq. (35). The final result is that

$$
\begin{aligned}
D_{r r}^{(\mu \nu)}= & -\tilde{D}_{\text {ret }}^{A}\left(\tilde{D}_{\text {ret }}^{A}\right)^{*} \\
& \times\left[\left\{\alpha|X|^{2}-2 \delta R \operatorname{Re}\left(X W^{*}\right)+\gamma R|W|^{2}\right\} P_{L}^{\mu \nu}\right. \\
& +\left\{\gamma|Z|^{2}-2 \delta R \operatorname{Re}\left(Z W^{*}\right)+\alpha R|W|^{2}\right\} C^{\mu \nu} \\
& +\left\{-\alpha \operatorname{Re}\left(X W^{*}\right)-\gamma \operatorname{Re}\left(Z W^{*}\right)\right. \\
& \left.\left.+\delta \operatorname{Re}\left(X Z^{*}\right)+\delta R|W|^{2}\right\} D^{\mu \nu}\right]-\tilde{D}_{\mathrm{ret}}^{B}\left(\tilde{D}_{\mathrm{ret}}^{B}\right)^{*} \beta E^{\mu \nu}
\end{aligned}
$$

where

$$
\begin{aligned}
X & =Q^{2}-\Pi_{c}, \\
Z & =Q^{2}-\Pi_{L}, \\
W & =-\Pi_{d}
\end{aligned}
$$

and

$$
\tilde{D}_{\text {ret }}^{A}=\frac{1}{\left(Q^{2}-\Pi_{L}\right)\left(Q^{2}-\Pi_{c}\right)-R \Pi_{d}^{2}}
$$

and

$$
\tilde{D}_{\text {ret }}^{B}=\frac{1}{Q^{2}-\Pi_{e}}
$$

with $R=Q^{2} \mathbf{q}^{2} /\left(q^{0}\right)^{2}$. Further details of this derivation can be found in Appendix A. Previously, the 00 component of the $r r$ correlator had been derived [51]. 


\section{INSTABILITIES IN AN ANISOTROPIC PLASMA}

Having calculated the density of soft gluons in an anisotropic medium, Eq. (38), we expect to obtain the rate of transverse momentum broadening from Eq. (14). However, this gives a divergent collision kernel $\mathcal{C}\left(\mathbf{q}_{\perp}\right)$ (see Appendix B for the mathematical details). The divergence is due to instabilities that are always present in an anisotropic plasma. The physical origin of these instabilities is widely discussed in the literature (see [52,53] for an early discussion, as well as [16,54,55] for a review). In essence, hard quasiparticles spontaneously break into filaments of currents which source soft chromomagnetic fields. These soft fields deflect the hard quasiparticles which makes the currents even stronger. This sources even stronger soft gluon fields, and so on, leading to an exponential growth in the density of soft gluons.

The divergence in the collision kernel forces us to examine implicit assumptions we made in Sec. III. First, we assumed a slowly changing medium. This means that the momentum distribution of hard quasiparticles, which appear in bare propagators, remains constant during jet momentum broadening. Second, we specified these momentum distributions at time $t_{0}=-\infty$. This made Fourier transforms possible, leading to simple expressions such as the self-energies in Eqs. (22) and (24) in momentum space.

Specifying an initial condition at time $t_{0}=-\infty$ might seem justified in a slowly changing medium. However, this assumption is invalidated by instabilities. In a strict HTL regime, the density of soft gluons continues to grow from the initial time due to instabilities. Thus at the time of a gluon kick on a jet parton, the density of gluons will have grown to be infinite and the rate of momentum broadening diverges. ${ }^{7}$

The solution to this problem is to specify the initial momentum distribution at a finite time, $t_{0}=0$, and to take into account its time evolution. Our recent work [15] did this analytically by deriving the evolution of the $r r$ correlator in an anisotropic system. We assumed a small anisotropy in the momentum distribution of hard particles, $\xi^{3 / 2} \ll g^{2}$, where

$$
\xi \sim \frac{\left|\left\langle p_{z}\right\rangle-\left\langle p_{\perp}\right\rangle\right|}{\left\langle p_{z}\right\rangle}
$$

This small anisotropy is needed for an adiabatic approximation. Specifically, the growth rate of instabilities is $\gamma \sim$ $\xi^{3 / 2} g \Lambda$ [56] and we need the growth rate to be slower than the process of interest.

\footnotetext{
${ }^{7}$ This problem of a divergent rate due to instabilities is not unique to momentum broadening. All probes that depend on the density of soft gluons suffer from the same spurious divergences in an anisotropic medium with initial condition specified at time $t_{0}=-\infty$. This includes the rate of photon production through bremsstrahlung, medium-induced jet splitting, and medium-induced quasiparticle splitting [8], which all depend on momentum broadening. Furthermore, the imaginary part of the heavy quark potential also diverges under these assumptions [51].
}

Assuming a small anisotropy, $\xi \ll 1$, the retarded correlator is

$$
G_{\text {ret }}\left(t_{x}, t_{y} ; \mathbf{p}\right)=\int_{\alpha} \frac{d p^{0}}{2 \pi} e^{-i p^{0}\left(t_{x}-t_{y}\right)} G_{\text {ret }}\left(p^{0}, \mathbf{p}\right)
$$

shortly after initialization in our setup when the HTL approximation is still valid. Here $G_{\text {ret }}\left(p^{0}, \mathbf{p}\right)=\left[\left(G_{\text {ret }}^{0}(P)\right)^{-1}-\right.$ $\left.\Pi_{\text {ret }}(P)\right]^{-1}$ where $\alpha$ is a contour that goes above all poles (see [15]), and the self-energy is given by Eq. (24). In particular it goes above poles $\omega=i \gamma$ with $\gamma>0$ that correspond to instability modes and lie in the upper half of the complex plane in $\omega$ [43]. Such an instability pole gives exponential growth in the time domain where $G_{\mathrm{ret}}\left(t_{x}, t_{y}\right) \sim \theta\left(t_{x}-t_{y}\right) e^{\gamma\left(t_{x}-t_{y}\right)}$.

Some earlier work on energy loss in an anisotropic plasma [37,38] implicitly assumed an integration contour along the real axis. This gives an incorrect contribution $\theta\left(t_{y}-\right.$ $\left.t_{x}\right) e^{\gamma\left(t_{x}-t_{y}\right)}$ to the retarded propagator in the time domain and leads to incorrect results for energy loss. We discuss this further in Appendix D.

In [15] we furthermore used a separation of scales to evaluate the $r r$ correlator in a system initialized at time $t_{0}=0$. We write the retarded correlator in momentum space as

$$
G_{\text {ret }}(K)=\widehat{G}_{\text {ret }}(K)+\sum_{i} \frac{A_{i}}{k^{0}-i \gamma_{i}}
$$

where $i \gamma_{i}$ are all poles of order $\xi^{3 / 2} g \Lambda$ including instability poles and $\widehat{G}_{\text {ret }}$ describes modes of order $g \Lambda .^{8}$ Then

$$
\begin{aligned}
G_{r r}\left(t_{x}, t_{y} ; \mathbf{k}\right) \approx & \int \frac{d k^{0}}{2 \pi} e^{-i k^{0}\left(t_{x}-t_{y}\right)} \widehat{G}_{r r}(K) \\
& +\sum_{i, j} \frac{A_{i} \Pi_{a a}(0) A_{j}^{*}}{\gamma_{i}+\gamma_{j}}\left[e^{\gamma_{i} t_{x}} e^{\gamma_{j} t_{y}}-1\right]
\end{aligned}
$$

shortly after initialization in our setup where

$$
\widehat{G}_{r r}(K)=\widehat{G}_{\mathrm{ret}}(K) \Pi_{a a}(K) \widehat{G}_{\mathrm{adv}}(K)
$$

and $\widehat{G}_{\mathrm{adv}}=\widehat{G}_{\mathrm{ret}}^{*}$. Modes of order $g \Lambda$ are described by $\widehat{G}_{r r}$. These fluctuating soft modes are continuously sourced by hard particles in the medium and they only depend on the particles' instantaneous momentum distribution $f(\mathbf{p})$, given that the HTL approximation is valid. Furthermore, their density does not depend on how much time has passed since initialization. Instability modes at energy $\xi g \Lambda$ are described by the second term. They grow exponentially in time shortly after initialization. ${ }^{9}$

Equation (47) for the $r r$ correlator in an anisotropic plasma is only valid for the first instants after a system is initialized. It corresponds to the early times in classical-statistical simulations of heavy-ion collisions $[17,18]$. However, we are

\footnotetext{
${ }^{8}$ The power in $\xi^{3 / 2} g \Lambda$ is for example derived in [56]. More generally, we are separating into modes with frequency $\sim g \Lambda$ and modes with frequency $\ll g \Lambda$.

${ }^{9}$ Further discussion can be found in Appendix E. In particular, we correct some wrong signs in a heuristic discussion of the $r r$ propagator in an unstable plasma in our earlier paper [15]. These sign mistakes in no way change the results of that paper.
} 


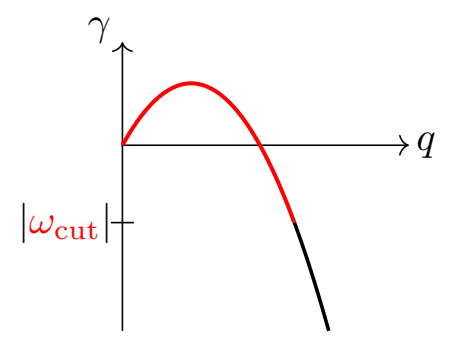

FIG. 2. Definition of $\omega_{\text {cut }}$. Instability poles $i \gamma$ are plotted with momentum $q$ for a particular direction. All poles in red are subtracted, i.e., all poles in the upper half plane, as well as all poles in the lower half plane with $|\gamma|<\omega_{\text {cut }}$.

interested in the collision kernel at later stages of heavy-ion collisions where instability modes have been saturated and the system has achieved a HTL-like scale separation after going through a nonthermal fixed point [19]. During these later stages, namely, the kinetic theory stage and hydrodynamics stage, the part of (47) describing fluctuating modes remains valid: Those gluons are continuously sourced by the hard quasiparticles in the medium and their density does not depend on the history of the medium. On the other hand, the ultrasoft instability modes at scale $\xi^{3 / 2} g \Lambda$ have evolved through self-interaction which is not captured by our analytic calculation.

In this paper, we focus on momentum broadening due to the fluctuating modes. More precisely, we choose a scale $\omega_{\text {cut }}$ separating the fluctuating modes at energy $g \Lambda$ and the instability modes at energy $\xi^{3 / 2} g \Lambda$ with

$$
\xi^{3 / 2} g \Lambda \ll \omega_{\text {cut }} \ll g \Lambda \text {. }
$$

We will focus on modes at $\omega>\omega_{\text {cut }}$ which are captured by the HTL $r r$ correlator in Eq. (38). Conversely, we will not include the effect of ultrasoft modes, including instability modes, at $\omega<\omega_{\text {cut }}$, as their dispersion relation in heavy-ion collisions is not captured by our analytic calculation. These ultrasoft modes only occupy a small portion of momentum space and should give a limited contribution to momentum broadening, except for potential divergences. We explore this further below.

In practice, we locate instability poles numerically and subtract their contribution from the retarded and advanced correlators. These poles are only present for small momenta, $q \sim \xi^{1 / 2} g \Lambda$. We subtract all instability poles in the upper half complex plane which correspond to exponential growth and which should not be treated in momentum space. We furthermore subtract poles in the lower half plane with $\omega=i \gamma$ and $|\gamma|<\omega_{\text {cut }}$ (see Fig. 2). These modes are on the second Riemann sheet in [57] which appears when the branch cut corresponding to Landau damping is modified [15]. ${ }^{10}$ This gives an $r r$ correlator for fluctuating modes

$$
\widehat{G}_{r r}(K)=\widehat{G}_{\mathrm{ret}}(K) \Pi_{a a}(K) \widehat{G}_{\mathrm{adv}}(K)
$$

\footnotetext{
${ }^{10}$ Modifying the branch cut does not change the correlator in the time domain where it is properly defined (see [58]).
}

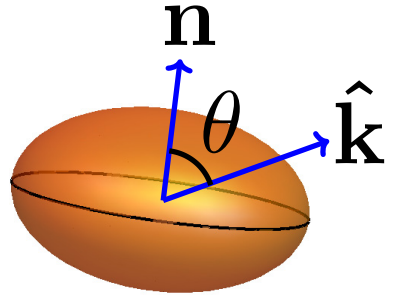

FIG. 3. The jet parton direction is defined through $\theta$, the angle between the jet momentum $\hat{\mathbf{k}}$ and the anisotropy vector $\mathbf{n}$. Pictured is the case of $\xi>0$ where the momentum distribution is oblate. For $\xi<0$ the distribution is prolate.

which coincides with that of Eq. (38) except that ultrasoft instability poles are subtracted in the retarded and advanced correlator. For numerical convenience we perform the subtraction of a pole $\omega=i \gamma$ in $\tilde{D}_{\text {ret }}^{A}$ and $\tilde{D}_{\text {ret }}^{B}$ from Eqs. (42) and (43). Defining $\tilde{D}_{\text {ret }}^{A}=1 / A(\omega)$, we write exactly

$$
\begin{aligned}
\frac{1}{A(\omega)} & =\frac{1}{\frac{A(\omega)}{(\omega-i \gamma)}(\omega-i \gamma)} \\
& =\frac{1}{A(\omega)-\left(\frac{A(\omega)}{\omega-i \gamma}\right)^{2}}-\frac{1}{(\omega-i \gamma)-\frac{A(\omega)}{\omega-i \gamma}} \frac{1}{\omega-i \gamma}
\end{aligned}
$$

and subtract the second term which has a pole at $\omega=i \gamma$ and the correct residue as $\omega \approx i \gamma$. The first term has no pole at $\omega=i \gamma$ because

$$
A(\omega)-\left(\frac{A(\omega)}{\omega-i \gamma}\right)^{2}=\frac{A(\omega)}{\omega-i \gamma}\left[\omega-i \gamma-\frac{A(\omega)}{\omega-i \gamma}\right]
$$

where $\frac{A(\omega)}{\omega-i \gamma}$ is finite everywhere and nonzero at $\omega=i \gamma$.

\section{RESULTS}

\section{A. Dependence on $\omega_{\text {cut }}$}

The collision kernel $\mathcal{C}\left(\mathbf{p}_{\perp}\right)$ depends on both medium properties and the direction of the jet. Specifically, the kernel depends on the anisotropy $\xi$ of the medium, the jet direction $\theta$, the magnitude of the transverse momentum kick $p_{\perp}$, and its direction $\phi$. The medium anisotropy $\xi$ is defined in Eq. (20) for the momentum distribution of quasiparticles. It describes elongation or contraction of an isotropic distribution in a direction $\mathbf{n}$. The direction of the jet is specified by $\theta$, the angle between the jet momentum and $\mathbf{n}$ (see Fig. 3). The symmetry of our setup means that only values of $0 \leqslant \theta \leqslant \pi / 2$ need to be considered. The direction of a momentum kick in the plane transverse to the jet momentum is given by an angle $\phi$. We choose $\phi=0$ when $\mathbf{p}_{\perp}$ is in the plane defined by the jet direction and $\mathbf{n}$.

We have obtained consistent expressions for the collision kernel by subtracting ultrasoft instability poles at frequency below a cut $\omega_{\text {cut }}$, leaving fluctuating modes at energy $\omega>\omega_{\text {cut }}$ which are the focus of this paper. It is important to explore how sensitive our results are to the exact value of the cut. ${ }^{11}$

\footnotetext{
${ }^{11}$ To subtract instability poles in the lower half plane on the second Riemann sheet, one needs the analytic continuation of the propagator
} 

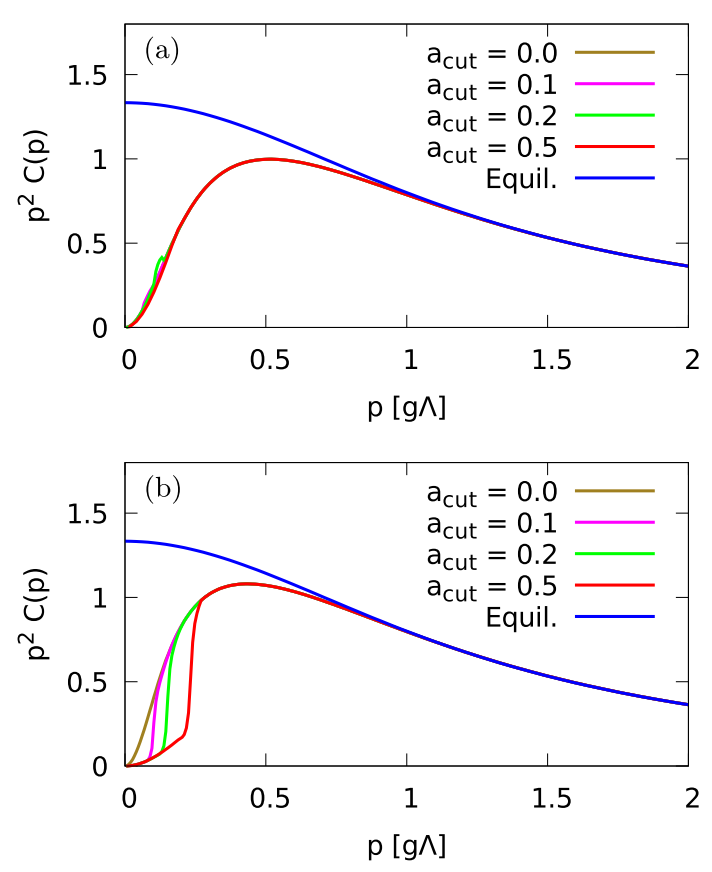

FIG. 4. The amount of transverse momentum broadening $\mathbf{p}_{\perp}^{2} \mathcal{C}(\mathbf{p})$ at momentum $\mathbf{p}_{\perp}$ in units of $g^{2} \Lambda$. (a) Anisotropy $\xi=-0.1$, jet direction $\theta=\pi / 2$, angle in transverse plane $\phi=0$. (b) $\xi=-0.1$, $\theta=\pi / 3, \phi=0$. The transverse momentum $\mathbf{p}_{\perp}$ is measured in units of $g \Lambda$. The different curves have different $\omega_{\text {cut }}=a_{\text {cut }} \xi^{3 / 2} g \Lambda$, the cut between fluctuating modes and ultrasoft instability modes which are subtracted. The equilibrium result with temperature $T=\Lambda$ and $\xi=0$ is shown for comparison. The dependence on the cut is mild for these values of $\xi$ and $\theta$. A similar picture holds in general for $\xi<0$ at $\pi / 4<\theta \leqslant \pi / 2$, and for $\xi>0$ at $0 \leqslant \theta<\pi / 4$.

This measures the robustness of our results and the importance of ultrasoft modes in momentum broadening. We will write $\omega_{\text {cut }}=a_{\text {cut }} \xi^{3 / 2} g \Lambda$ where $a_{\text {cut }}$ is a number. ${ }^{12}$ All our results will assume a QCD plasma with three flavors of massless quarks.

The sensitivity of momentum broadening on the cut $\omega_{\text {cut }}$ is moderate and depends on the direction of the jet: in Figs. 4 and 5 we show the amount of transverse broadening at momentum $\mathbf{p}_{\perp}$ as the cut is varied. ${ }^{13}$ These figures are representative for the results at a negative anisotropy, $\xi<0$. For jet direction $\pi / 4 \leqslant \theta \leqslant \pi / 2$ as in Fig. 4, there is limited dependence on the cut and one gets consistent results by subtracting the

to that Riemann sheet. The self-energies in Eq. (32) have one remaining integral and thus cannot be analytically continued in any simple way. Instead, we will use the expansion at low $\xi$ for $\Pi_{\text {ret }}$ derived in $[43,48]$ when estimating the effect of $\omega_{\text {cut }}$ in Figs. 4 and 5. As we subsequently choose $\omega_{\text {cut }}=0.0$, with no poles in the lower half plane subtracted, we use the full results valid at all $\xi$ in all other figures.

${ }^{12}$ For comparison the maximal growth rate from instabilities in $\tilde{D}_{\text {ret }}^{A}$ in Eq. (42) is $\gamma_{\max } \approx 0.15 g \Lambda \xi^{3 / 2}$ [48]. Thus a reasonable choice for the cut in the lower half plane is $a_{\text {cut }} \approx 0.1-0.5$.

${ }^{13}$ Subtracting poles with $|\gamma|<\omega_{\text {cut }}$ introduces kinks in $\mathcal{C}\left(\mathbf{p}_{\perp}\right)$ (see Fig. 5). Including the physics of ultrasoft modes would give a continuous description and remove these kinks.
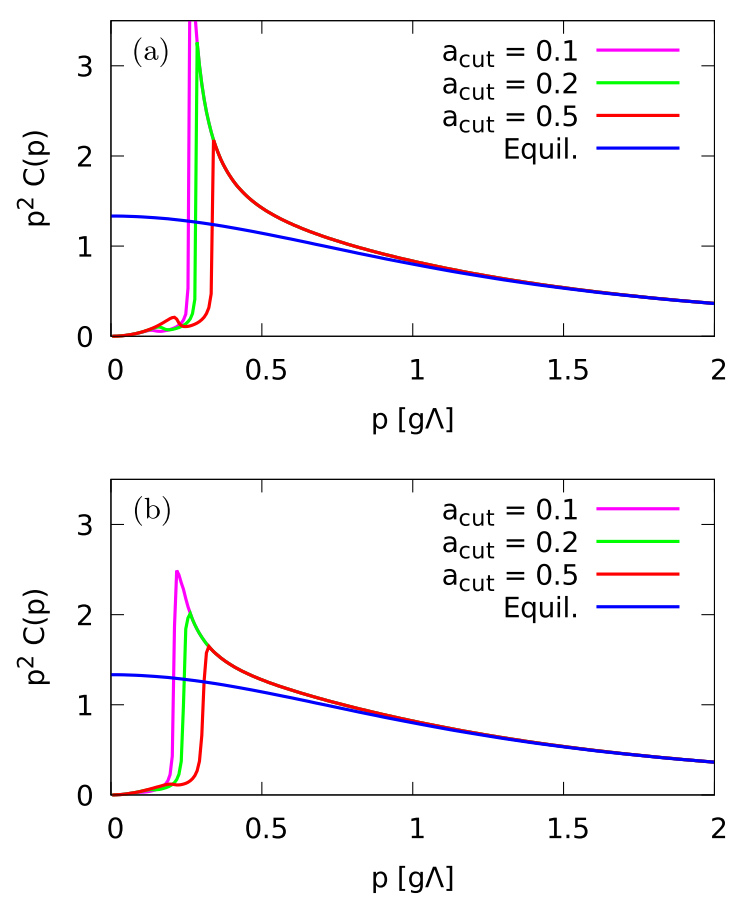

FIG. 5. The dependence of transverse momentum broadening on $\omega_{\text {cut }}=a_{\text {cut }} \xi^{3 / 2} g \Lambda$ for two values of $\theta$. (a) $\xi=-0.1, \theta=0, \phi=0$. (b) $\xi=-0.1, \theta=\pi / 6, \phi=0$. The dependence on the cut is more pronounced than in Fig. 4 due to slowly decaying modes transverse to the jet direction. A similar picture holds in general for $\xi<0$ at $0 \leqslant \theta<\pi / 4$ and for $\xi>0$ at $\pi / 4 \leqslant \theta \leqslant \pi / 2$.

instability poles. For $0 \leqslant \theta<\pi / 4$ as in Fig. 5 there is more dependence on the value of the cut.

The different qualitative behavior in Figs. 4 and 5 can be understood by analyzing the structure of instability poles. For negative anisotropy, when $\pi / 4 \leqslant \theta \leqslant \pi / 2$ there are instability poles in $D_{\text {ret }}^{A}$ with momentum transverse to the direction of the jet [48]. Even after subtracting the poles below $\omega_{\text {cut }}$ there remain very slowly decaying modes with $\omega=-i \gamma, \gamma \gtrsim \omega_{\text {cut }}$ which have momentum nearly transverse to the jet direction. Due to the slow decay of these modes, each excitation can impart transverse momentum to the jet for a long time. Thus these modes can impart a great deal of transverse momentum which explains the rapid rise in Fig. 5. As there are no such slowly decaying modes transverse to the jet direction for $0 \leqslant \theta<\pi / 4$ one gets much less sensitivity to the cut. ${ }^{14}$ The picture is opposite for positive anisotropy. Due to the location of instability poles, one gets little sensitivity to the cut for $0 \leqslant \theta<\pi / 4$ like in Fig. 4 and more sensitivity for $\pi / 4 \leqslant \theta \leqslant \pi / 2$ like in Fig. 5 .

\footnotetext{
${ }^{14}$ For these values of $\theta$, one would get a convergent result without a cutoff. However, such a calculation would not be physical as it would treat exponentially growing modes incorrectly. Specifically, it would assume that the contribution of the instability modes to the retarded correlator $D_{\text {ret }}\left(t_{x}, t_{y}\right)$ was at $t_{y}>t_{x}$, which is not in accordance with properties of the retarded propagator. (See Appendix C for a discussion on this in the case of heavy quarks.)
} 


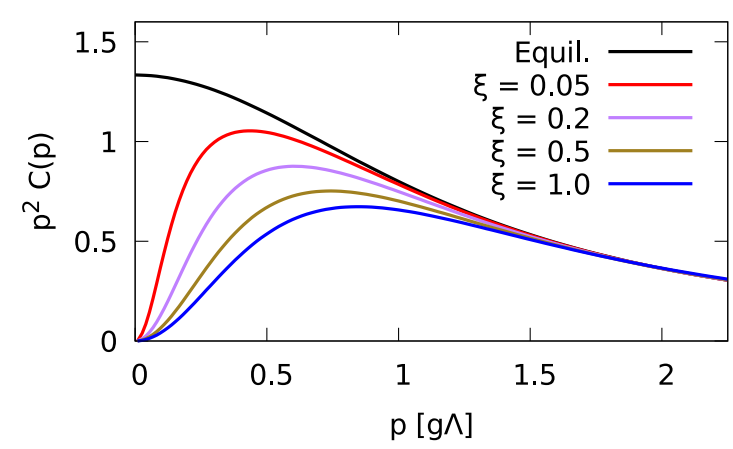

FIG. 6. Transverse momentum broadening for a jet parton parallel to the anisotropy vector, $\theta=0$, for positive anisotropy. Momentum broadening is reduced as the anisotropy increases, especially for low and intermediate values of $\mathbf{p}_{\perp}$. The hard scale of the problem $\Lambda$ is roughly the temperature of the medium. Its values in heavy-ion collisions are in the range of roughly 150 and $300 \mathrm{MeV}$.

The dependence of $\widehat{q}$ on the frequency cut can be estimated analytically (see Appendix B). One gets that

$$
\widehat{q} \sim g^{4} \Lambda^{3} \xi^{3 / 2} \log \left(\sqrt{\xi} \omega_{\text {cut }}\right) .
$$

This relatively mild dependence is because of the small phase space in which one finds instabilities.

For the rest of this paper we will focus on values of $\xi$ and $\theta$ where there is less sensitivity to the cut, like in Fig. 4. For simplicity we will choose $\omega_{\text {cut }}=0.0$. For other values of $\xi$ and $\theta$ like in Fig. 5, the collision kernel is sensitive to the exact dispersion relation and occupation density of ultrasoft modes. Thus our calculation should eventually be complemented by detailed information on the far infrared in heavy-ion collisions. This would of course cancel any dependence on $\omega_{\text {cut }}$ in our calculation.

\section{B. Dependence on anisotropy and jet direction}

Medium anisotropy reduces momentum broadening because medium screening is increased. In Fig. 6 momentum broadening is shown for different positive values of $\xi$ where the jet parton is parallel to the anisotropy vector, $\theta=0$. In Fig. 7 momentum broadening is shown for negative values of

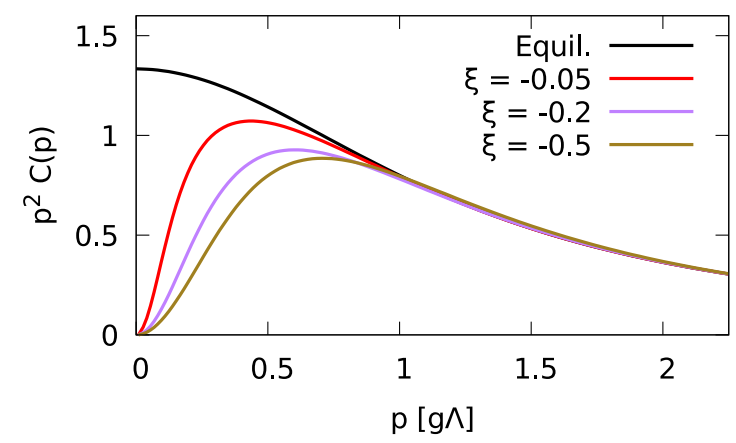

FIG. 7. Transverse momentum broadening for a jet parton transverse to the anisotropy vector, $\theta=\pi / 2$, for negative anisotropy. As in Fig. 6, momentum broadening is reduced in a more anisotropic medium. Here $\phi=0$.

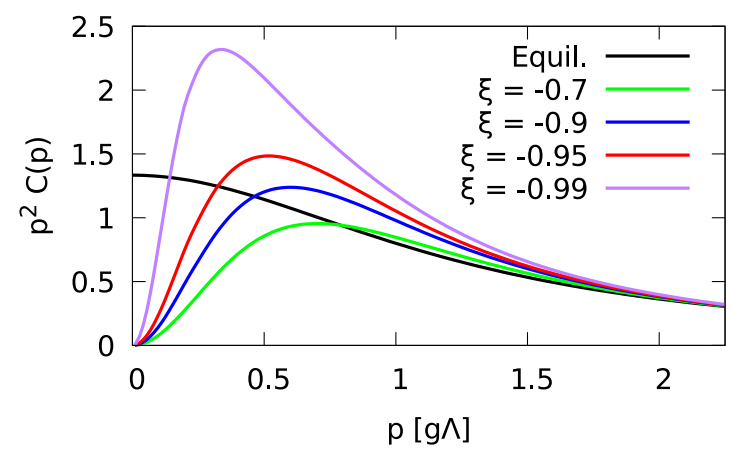

FIG. 8. Transverse momentum broadening at high negative values of $\xi$ with $\theta=\pi / 2$ and $\phi=0$. An additional peak develops at $p_{\perp} \sim 0.5 g \Lambda$ relative to low and intermediate values of $\xi$ as in Fig. 7 . This regime of extreme anisotropy stretches our assumption that modes can be separated into instability modes and anisotropy modes.

$\xi$ with the jet transverse to the anisotropy vector, $\theta=\pi / 2$. Both results are qualitatively similar. Deviation from equilibrium universally results in less momentum broadening. ${ }^{15}$ This reduction is clear even at small anisotropy. The deviations from equilibrium are most pronounced at low and intermediate values of $\mathbf{p}_{\perp}$ as at high values medium screening is unimportant. We have also checked the dependence on the angle $\theta$ between the jet parton and the anisotropy vector. It is found to be fairly mild.

The qualitative difference between equilibrium and nonequilibrium momentum broadening is due to additional screening in the nonequilibrium plasma. The equilibrium collision kernel is $\mathcal{C} \sim 1 / \mathbf{q}_{\perp}^{2}$ for low transverse momentum as can be seen in Eq. (19). This is because of the absence of static screening for magnetic modes in a quark-gluon plasma in thermal equilibrium [2] while the electric mode gets screening from the Debye mass $m_{D}^{2}$. Conversely, our nonequilibrium collision kernel goes to a constant at very low $\mathbf{q}_{\perp}$. This is a sign of additional screening in a nonequilibrium medium. In particular, the self-energy component $\Pi_{c}$ goes to zero in thermal equilibrium in the limit $\omega \rightarrow 0$, denoting absence of magnetic screening, but remains finite in the anisotropic case. This is true even for moderate values of the instability cutoff $\omega_{\text {cut }}$.

Our separation of physics into instability modes and fluctuating modes makes the most sense for low and intermediate values of $\xi$. It is nevertheless interesting to see how the collision kernel behaves at extreme anisotropy in our setup. In Fig. 8 we show the kernel for very large negative values of $\xi$ which must be bounded from below by -1 . At extreme anisotropy, the collision kernel develops a pronounced peak around $p_{\perp}=0.5 g \Lambda$.

A nonequilibrium collision kernel is needed to calculate the rate of quasiparticle one-to-two radiation in kinetic theory

\footnotetext{
${ }^{15}$ This is universally true for values of $\xi$ and $\theta$ like in Fig. 4 which have little sensitivity to $\omega_{\text {cut }}$. For values of $\xi$ and $\theta$ where information on ultrasoft modes is required like in Fig. 5, it is not clear to us whether the same reduction in momentum broadening will take place.
} 


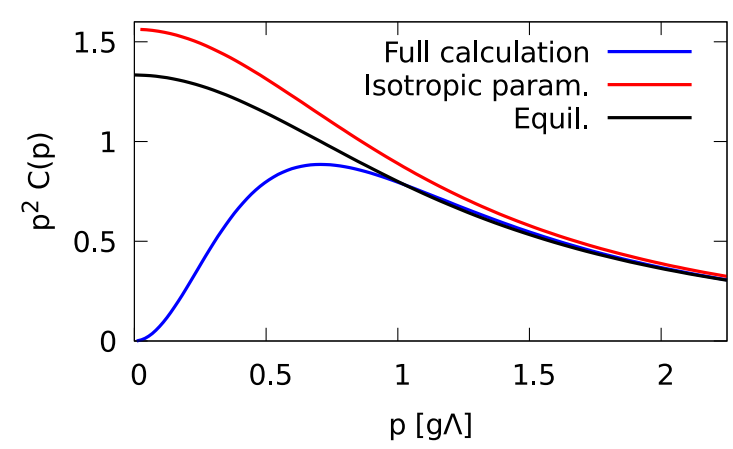

FIG. 9. Comparison between the isotropic parametrization used in effective kinetic theory and our anisotropic collision kernel for $\xi=$ -0.5 and $\theta=\pi / 2$. The isotropic parametrization does not capture the anisotropic behavior and incorrectly shows increased momentum broadening. The comparison for other values of $\xi$ and $\theta$ is similar.

[8]. In the absence of an anisotropic nonequilibrium collision kernel, kinetic theory simulations have used an isotropic parametrization $[11,12]$ which is exact in an isotropic medium and is given by Eq. (19) where the equilibrium Debye mass is replaced by

$$
m_{D}^{2}=2 \int \frac{d^{3} p}{(2 \pi)^{3} p}\left[2 N_{f} f_{q}(\mathbf{p})+2 N_{c} f_{g}(\mathbf{p})\right]
$$

and the temperature is replaced by an effective temperature

$$
T_{*}=\frac{\frac{1}{2} \int \frac{d^{3} p}{(2 \pi)^{3}}\left\{2 N_{f} f_{q}(\mathbf{p})\left[1-f_{q}(\mathbf{p})\right]+2 N_{c} f_{g}(\mathbf{p})\left[1+f_{g}(\mathbf{p})\right]\right\}}{\int \frac{d^{3} p}{(2 \pi)^{3} p}\left[2 N_{f} f_{q}(\mathbf{p})+2 N_{c} f_{g}(\mathbf{p})\right]}
$$

(see [8]). In Fig. 9, we compare this parametrization, as well as the equilibrium collision kernel at temperature $T=\Lambda$, with our full anisotropic calculation of the collision kernel. Clearly, the isotropic parametrization does not capture the behavior of the collision kernel in an anisotropic medium. Including a full anisotropic collision kernel could affect the rate of quasiparticle splitting in kinetic theory simulations, and this influences the entire space-time evolution.

\section{Angular dependence of $\hat{\boldsymbol{q}}$}

In an anisotropic medium, momentum broadening is distorted with more broadening in one direction of the transverse plane than the other. In other words, the collision kernel $\mathcal{C}\left(\mathbf{q}_{\perp}\right)$ depends on the direction of $\mathbf{q}_{\perp}$. One way to quantify the total transverse momentum broadening in a particular direction is to consider

$$
\widehat{q}_{i j}=\int \frac{d^{2} q_{\perp}}{(2 \pi)^{2}} q_{\perp i} q_{\perp j} \mathcal{C}\left(\mathbf{q}_{\perp}\right) .
$$

There always exist orthogonal principal axes so that $\widehat{q}_{x y}=0$. In our case, one axis is in the plane spanned by the jet direction and the anisotropy vector, with the other one being orthogonal. The total transverse momentum broadening in Eq. (13) is given by $\widehat{q}=\widehat{q}_{x x}+\widehat{q}_{y y}$ and in general $\widehat{q}_{x x} \neq \widehat{q}_{y y}$.

Equation (56) is UV divergent and needs a cutoff. This cutoff depends on the process being considered. As an ex-
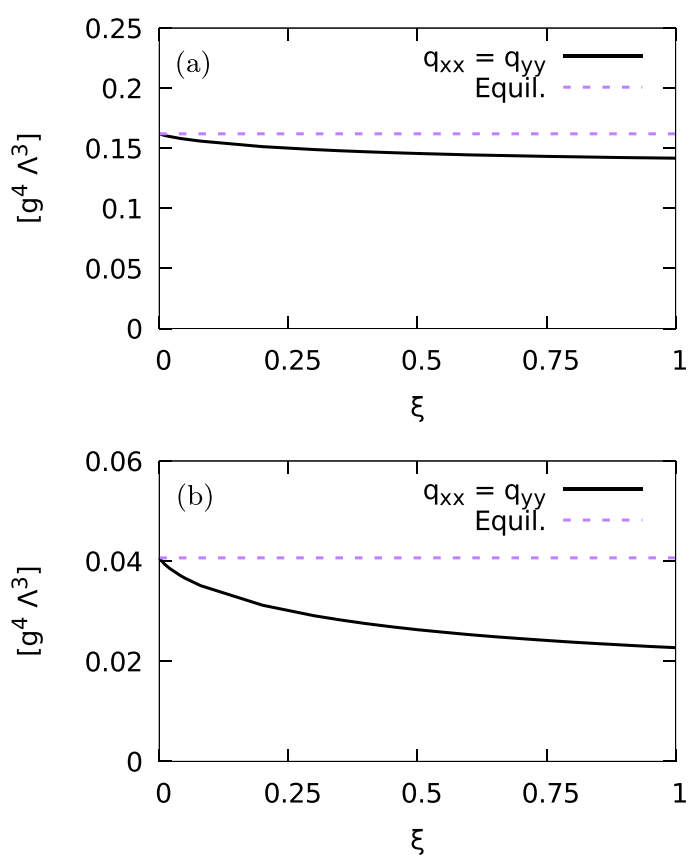

FIG. 10. The transport coefficients $q_{x x}$ and $q_{y y}$ in units of $g^{4} \Lambda^{3}$ in a medium with positive anisotropy. The jet parton travels parallel to the medium anisotropy, $\theta=0$. (a) UV cutoff of $q_{\max }=3.2 \mathrm{~g} \Lambda$ corresponding roughly to jet energy $E \sim 100 \Lambda$. (b) $q_{\max }=g \Lambda$, corresponding roughly to $E \sim \Lambda$.

ample the cutoff for radiation off a highly energetic jet is roughly

$$
q_{\max } \sim g \Lambda(E / \Lambda)^{1 / 4}
$$

where $E$ is the energy of the jet parton [59] and $\Lambda$ is the medium scale. This scaling relation becomes progressively better as the energy of the jet parton increases.

In Fig. 10, we show transverse momentum broadening for a parton that travels in the direction of the medium anisotropy, i.e., $\theta=0$. In this case the transverse plane is the same in all directions and $\widehat{q}_{x x}=\widehat{q}_{y y}$. We consider a medium with positive anisotropy. As the energy of a jet partons increases it receives transverse kicks of higher energy which are less sensitive to details of medium screening. Therefore, anisotropy has more effect on low-energy jet partons. As an example, for a jet parton with $E \sim 100 \Lambda$, the anisotropy gives a modest decrease of $15 \%$ to momentum broadening, while for medium particles or very low-energy jet partons with $E \sim \Lambda$, the decrease is nearly $45 \%$ (see Fig. 10).

In Fig. 11, we show transverse momentum broadening for a jet parton that travels orthogonally to the medium anisotropy, i.e., $\theta=\pi / 2$. We assume a medium with negative anisotropy. The effect of the anisotropy for a jet parton with energy $E \sim 100 \Lambda$ is small. However, for energy $E \sim \Lambda$ there is a decrease of around $20 \%$ for $q_{x x}$ and around $15 \%$ for $q_{y y}$ due to the anisotropy. Thus there is modest distortion in transverse broadening anisotropy.

The effects of anisotropy on momentum broadening are most substantial for low-energy particles such as medium particles. Therefore, the effects we have described should 

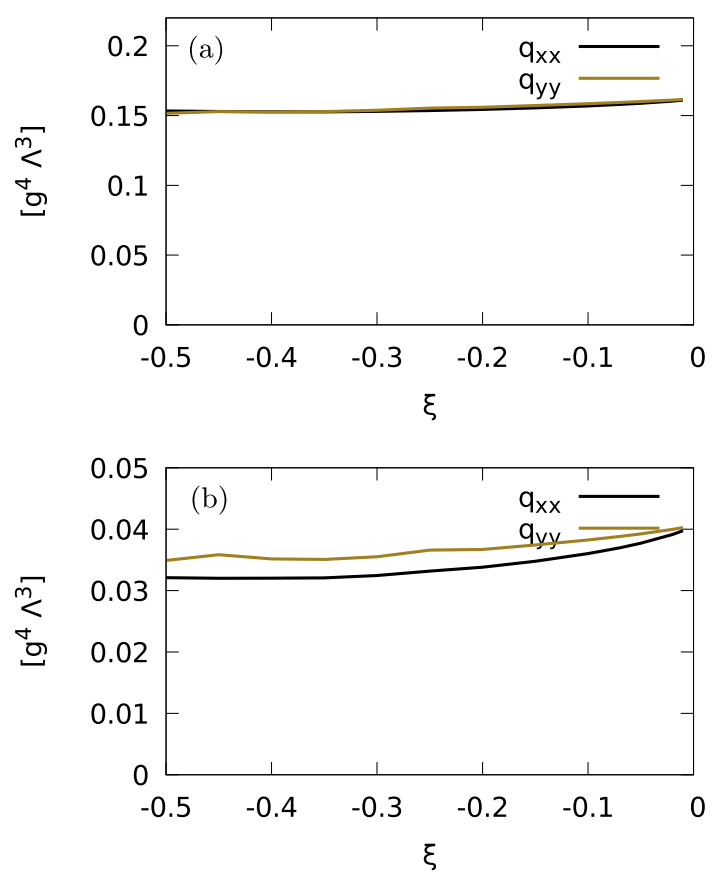

FIG. 11. The transport coefficients $q_{x x}$ and $q_{y y}$ in a medium with negative anisotropy. The jet parton is orthogonal to the medium anisotropy, $\theta=\pi / 2$. (a) $q_{\max }=3.2 g \Lambda$, corresponding roughly to $E \sim 100 \Lambda$. (b) $q_{\max }=g \Lambda$, corresponding roughly to $E \sim \Lambda$.

be particularly important for quasiparticle interaction in kinetic theory as well for photon radiation from a medium. In these cases a medium quark with $E \sim \Lambda$ receives transverse momentum kicks, causing it to radiate a gluon or a photon. Figures 10(b) and 11(b) give a rough indicator of how important the effect of anisotropy on momentum broadening could be for the rate of those processes. In particular, Fig. 10(b) suggests that the effect could be sizable. In this regime one must solve the full equations for rates of gluon and photon radiation derived in $[13,60]$ which require the full collision kernel $\mathcal{C}\left(\mathbf{q}_{\perp}\right)$. We will explore this further in a future publication.

\section{CONCLUSIONS}

The rate of transverse momentum broadening is an integral part of the physics of relativistic plasmas. This rate is given by the collision kernel $\mathcal{C}\left(\mathbf{q}_{\perp}\right)$, the probability that a jet parton gets transverse kicks of momentum $\mathbf{q}_{\perp}$ from the medium. The collision kernel also describes momentum broadening of a quasiparticle in the medium.

For the first time, we have calculated the collision kernel in a nonequilibrium plasma [see Eq. (14)]. This is motivated by the quark-gluon plasma in heavy-ion collisions, which is anisotropic and at times far from equilibrium. We assume an anisotropic momentum distribution of quasiparticles and calculate the $r r$ correlator of soft gluons in the medium [see Eq. (38)]. Even at small values of anisotropy, the collision kernel changes qualitatively from the equilibrium result (see Figs. 6 and 7). In those figures, additional screening leads to less momentum broadening, especially at small and interme- diate transverse momenta, an effect which is not captured by the isotropic ansatz used in kinetic theory simulations [11,12]. This gives a sizable decrease in the transport coefficient $\widehat{q}$, as well as mild angular dependence (see Figs. 10 and 11).

The collision kernel for momentum broadening determines the rate of medium-induced gluon radiation off a jet parton. It also determines the rate of one-to-two scattering of quasiparticles in a medium, as well as the rate of photon production through bremsstrahlung. With an anisotropic collision kernel in hand, we can therefore calculate consistently the rate of all these different processes in an anisotropic plasma. We will report on progress in a future publication.

A central challenge in this calculation is the presence of unstable modes in an anisotropic plasma. These modes lead to exponential growth in soft gluon density at very early times but numerical simulations suggest that at later times the modes become saturated in heavy-ion collisions [17-19]. This is not captured by our analytic calculation, leading us to subtract ultrasoft instability modes with exponential growth. Our focus is thus on fluctuating modes which are sourced at each instant by quasiparticles in the medium and the contribution of which to momentum broadening does not depend on the detailed history of the medium. However, we note that for certain values of the anisotropy and jet direction, the collision kernel is sensitive to the ultrasoft modes subtracted and thus our calculation needs to be complemented by a description of the deep infrared in heavy-ion collisions. An alternative would be to measure the collision kernel defined in terms of Wilson lines [61] directly from, say, classical-statistical simulations of heavy-ion collisions.

\section{ACKNOWLEDGMENTS}

This work was supported in part by the Natural Sciences and Engineering Research Council of Canada. S.H. gratefully acknowledges a scholarship from the Fonds de Recherche du Québec-Nature et Technologies.

\section{APPENDIX A: DERIVATION OF EQ. (38)}

In this Appendix we give further details for the derivation of Eq. (38). Combining Eqs. (36) and (37) we get that

$$
\begin{aligned}
D_{r r}^{(\mu \nu)}= & \frac{1}{2}\left[\left\{D_{\mathrm{ret}},\left\{-i \Pi_{a a}, D_{\mathrm{adv}}\right\}\right\}-\left\{-i \Pi_{a a},\left\{D_{\mathrm{adv}}, D_{\mathrm{ret}}\right\}\right\}\right. \\
& \left.+\left\{D_{\mathrm{adv}},\left\{D_{\mathrm{ret}},-i \Pi_{a a}\right\}\right\}\right]^{\mu \nu}
\end{aligned}
$$

where anticommutators act on Lorentz indices. The ingredients in this equation are the self-energy for gluon production,

$$
-i \Pi_{a a}^{\mu \nu}=\alpha P_{L}^{\mu \nu}+\beta E^{\mu \nu}+\gamma C^{\mu \nu}+\delta D^{\mu \nu},
$$

the retarded correlator

$$
\begin{aligned}
D_{\text {ret }}^{\mu \nu}= & \frac{-i Q^{\mu} Q^{\nu}}{\left(Q^{2}\right)^{2}}+i E^{\mu \nu} \tilde{D}_{\text {ret }}^{B} \\
& +i\left[X P_{L}^{\mu \nu}+Z C^{\mu \nu}-W D^{\mu \nu}\right] \tilde{D}_{\text {ret }}^{A},
\end{aligned}
$$


and the advanced correlator

$$
D_{\mathrm{adv}}^{\mu \nu}=\frac{-i Q^{\mu} Q^{\nu}}{\left(Q^{2}\right)^{2}}+i E^{\mu \nu} \tilde{D}_{\mathrm{ret}}^{B *}+i\left[X^{*} P_{L}^{\mu \nu}+Z^{*} C^{\mu \nu}-W^{*} D^{\mu \nu}\right] \tilde{D}_{\mathrm{ret}}^{A *} .
$$

The quantities $X, Z, W, \tilde{D}_{\text {ret }}^{A}$, and $\tilde{D}_{\text {ret }}^{B}$ can be found in Eqs. (39)-(43).

To evaluate the anticommutators in Eq. (A1), we need anticommutators of the tensors $P_{L}, E, C$, and $D$. These are given in Eq. (35). As an example, these relations can be used to show that

$$
\begin{aligned}
\left\{D_{\mathrm{adv}}, D_{\mathrm{ret}}\right\}^{\mu \nu} & =\left\{i E^{\mu \nu} \tilde{D}_{\text {ret }}^{B *}+i \tilde{D}_{\text {ret }}^{A *}\left(X^{*} P_{L}^{\mu \nu}+Z^{*} C^{\mu \nu}-W^{*} D^{\mu \nu}\right), i E^{\mu \nu} \tilde{D}_{\text {ret }}^{B}+i \tilde{D}_{\text {ret }}^{A}\left(X P_{L}^{\mu \nu}+Z C^{\mu \nu}-W D^{\mu \nu}\right)\right\}^{\mu \nu} \\
& =\left|\tilde{D}_{\text {ret }}^{B}\right|^{2} E^{\mu \nu}+\left|\tilde{D}_{\text {ret }}^{A}\right|^{2}\left(|X|^{2} P_{L}^{\mu \nu}+|Z|^{2} C^{\mu \nu}+|W|^{2} \frac{Q^{2} \mathbf{q}^{2}}{\left(q^{0}\right)^{2}}\left(C+P_{L}\right)^{\mu \nu}-2 \operatorname{Re}\left(X^{*} W\right) D^{\mu \nu}-2 \operatorname{Re}\left(Z^{*} W\right) D^{\mu \nu}\right)
\end{aligned}
$$

where we evaluated the anticommutators term by term. Using this result one can then evaluate the term $\left\{-i \Pi_{a a},\left\{D_{\text {adv }}, D_{\text {ret }}\right\}\right\}$ in Eq. (A1) using the same relations for anticommutators. A straightforward but tedious calculation of all the terms in Eq. (A1) then results in Eq. (38).

\section{APPENDIX B: ESTIMATE OF DEPENDENCE ON MOMENTUM CUTOFF}

We will now show in detail the logarithmic divergence that occurs due to instability modes when momentum broadening is evaluated as in Sec. III making the (incorrect) assumption of an initial condition at time $t_{0}=-\infty$. For simplicity, we will consider the case where the jet parton momentum $\widehat{\mathbf{k}}$ is parallel to the anisotropy vector $\mathbf{n}$ which we choose to be the $z$ axis. Momentum broadening is given by

$$
\widehat{q} \approx g^{2} C_{F} \int \frac{d^{4} Q}{(2 \pi)^{4}} q_{\perp}^{2} D_{r r}^{\mu \nu}(Q) v_{\mu} v_{\nu} \delta(v \cdot Q)
$$

where

$$
D_{r r}=D_{\text {ret }} \Pi_{a a} D_{\mathrm{adv}}
$$

in this naive setup. The blowup due to instabilities comes from the instability poles in $D_{\text {ret }}$ and $D_{\text {adv }}$. We focus on the instability poles coming from the term with $\Pi_{e}$. The other contribution can be evaluated similarly. Using results from $[37,48]$ one can show that at small anisotropy

$$
\begin{aligned}
\Pi_{e}= & m_{D}^{2}\left(\frac{\omega}{q}\right)^{2}-\frac{i \pi}{4} m_{D}^{2} \frac{\omega}{q}+O\left[\left(\frac{\omega}{q}\right)^{3}\right] \\
& +\xi\left[-\frac{1}{6}(1+\cos 2 \theta) m_{D}^{2}+O\left(\frac{\omega}{q}\right)\right] .
\end{aligned}
$$

We verify below that the expansion in $\omega / q \ll 1$ is consistent. This clearly shows the presence of instability poles as

$$
\omega^{2}=q^{2}+\Pi_{e} \approx q^{2}-\xi \frac{1}{6}(1+\cos 2 \theta) m_{D}^{2}
$$

has an imaginary solution $\omega=i \gamma$ for sufficiently small values of $q$. For our simple estimate we can ignore the dependence of the pole on the angle $\theta$ between the gluon momentum $\mathbf{q}$ and the anisotropy vector $\mathbf{n}$.
Ignoring all numerical factors and substituting $D_{\text {ret }} \rightarrow$ $1 /\left(Q^{2}-\Pi_{e}\right)$ and $D_{a d v}=D_{\text {ret }}^{*}$ in Eq. (B1), we get

$$
\begin{aligned}
\widehat{q} \sim g^{3} \Lambda^{2} \int d^{4} Q q_{\perp}^{2} \delta\left(\omega-q^{z}\right) \\
\quad \times \frac{1}{\left(q_{\perp}^{2}+m_{D}^{2}\left(\frac{\omega}{q}\right)^{2}-\xi m_{D}^{2}\right)^{2}+\left(m_{D}^{2} \frac{\omega}{q}\right)^{2}} .
\end{aligned}
$$

It is clear that this expression diverges when $q_{\perp} \sim \sqrt{\xi} m_{D}$ and $\omega \sim q_{\perp}^{3} / m_{D}^{2} \sim \xi^{3 / 2} m_{D}$, justifying our approximation that $\omega / q \ll 1$ in Eq. (B3) when the anisotropy is small. Rewriting gives

$$
\widehat{q} \sim g^{3} \Lambda^{2} \int d q^{z} \int d q_{\perp} \frac{q_{\perp}^{3}}{\left(\frac{m_{D} q^{z}}{\sqrt{\xi}}\right)^{2}+\left(q_{\perp}^{2}-\xi m_{D}^{2}\right)^{2}} .
$$

Scaling all variables by $m_{D}$ and introducing a cutoff $\delta$ defined by $q_{\perp} \approx q>\sqrt{\xi} m_{D}+\delta m_{D}$ gives that

$$
\widehat{q} \sim g^{4} \Lambda^{3} \int d x \int_{\sqrt{\xi}+\delta} d z \frac{z^{3}}{x^{2} / \xi+\left(z^{2}-\xi\right)^{2}} .
$$

The remaining integrals can be done analytically by a change of variables $x \rightarrow x / \sqrt{\xi}$ and $y=z^{2}-\xi$, and then going to radial coordinates. The result is that dependence of momentum broadening on the cutoff is

$$
\widehat{q} \sim g^{4} \Lambda^{3} \xi^{3 / 2} \log (\sqrt{\xi} \delta) .
$$

Our derivation of the logarithmic blowup in Eq. (B8) was done in a naive framework where the initial condition is at $t_{0}=-\infty$. However, this derivation also tells us about the case where the initial condition is specified at $t_{0}=0$ (see Sec. IV). When specifying a cut $\omega_{\text {cut }}$, below which instability poles are subtracted, the treatment for modes of energy greater than $\omega_{\text {cut }}$ is the same as in the derivation of Eq. (B8). Given a relation between the three-momentum cutoff $\delta$ and the frequency cutoff $\omega_{\text {cut }}$, we therefore see that the dependence on the frequency cutoff is

$$
\widehat{q} \sim g^{4} \Lambda^{3} \xi^{3 / 2} \log \left(\sqrt{\xi} \omega_{\text {cutoff }}\right) .
$$

\section{APPENDIX C: GLUON SELF-ENERGY IN AN ANISOTROPIC MEDIUM}

To evaluate the $r r$ correlator we need the self-energy $\Pi_{a a}$ given by Eq. (22). Doing a change of variables to $\tilde{p}=$ 
$p \sqrt{1+\xi(\mathbf{v} \cdot \mathbf{n})^{2}}$ shows that

$$
\Pi_{a a}^{\mu \nu}=2 \pi m_{D, \mathrm{eq}}^{2} \Lambda P^{\mu \nu}
$$

where the angular part is

$$
P^{\mu \nu}=\left.\int \frac{d \Omega}{4 \pi} v^{\mu} v^{\nu} \frac{\delta(\omega-\mathbf{v} \cdot \mathbf{q})}{\left(1+\xi(\mathbf{v} \cdot \mathbf{n})^{2}\right)^{3 / 2}}\right|_{v^{0}=v} .
$$

Furthermore,

$$
\begin{aligned}
m_{D, \text { eq }}^{2}= & \frac{g^{2}}{2 \pi^{2}} \int_{0}^{\infty} d \tilde{p} \tilde{p}^{2} \\
& \times\left[2 N_{f} f_{q}^{0}\left(1-f_{q}^{0}\right)+2 N_{c} f_{g}^{0}\left(1+f_{g}^{0}\right)\right]
\end{aligned}
$$

is the Debye mass in thermal equilibrium with $f_{q}^{0}$ and $f_{g}^{0}$ the equilibrium distributions with the temperature substituted by $\Lambda$. Using the delta function in Eq. (C2) we get that

$$
\begin{aligned}
P^{00}= & \frac{1}{4 \pi q} \int_{0}^{2 \pi} d \phi \\
& \times \frac{1}{\left[1+\xi\left(\tilde{q}_{\|} \tilde{\omega}-\tilde{q}_{\perp} \sqrt{1-\tilde{\omega}^{2}} \cos \phi\right)^{2}\right]^{3 / 2}}, \\
P^{0 i} n^{i}= & \frac{1}{4 \pi q} \int_{0}^{2 \pi} d \phi \\
& \times \frac{1}{\left[1+\xi\left(\tilde{q}_{\|} \tilde{\omega}-\tilde{q}_{\perp} \sqrt{1-\tilde{\omega}^{2}} \cos \phi\right)^{2}\right]^{3 / 2}}, \\
P^{i j} n^{i} n^{j}= & \frac{1}{4 \pi q} \int_{0}^{2 \pi} d \phi \\
& \times \frac{\left(\tilde{q}_{\|} \tilde{\omega}-\tilde{q}_{\perp} \sqrt{1-\tilde{\omega}^{2}} \cos \phi\right)^{2}}{\left[1+\xi\left(\tilde{q}_{\|} \tilde{\omega}-\tilde{q}_{\perp} \sqrt{1-\tilde{\omega}^{2}} \cos \phi\right)^{2}\right]^{3 / 2}},
\end{aligned}
$$

where $\tilde{q}_{\|}=\mathbf{n} \cdot \mathbf{q} / q$ is the normalized component of the gluon momentum that is parallel to the anisotropy vector $\mathbf{n}, \tilde{q}_{\perp}=$ $|\mathbf{q}-(\mathbf{n} \cdot \mathbf{q}) \mathbf{n}| / q$ is the normalized transverse component, and $\tilde{\omega}=\omega / q$. We do the remaining integral numerically. See [45] for an alternative evaluation, partially in terms of special functions.

We can finally assemble the components of $\Pi_{a a}$ found in Eq. (30):

$$
-i \Pi_{a a}=\alpha P_{L}+\beta E+\gamma C+\delta D
$$

They are

$$
\begin{aligned}
i \alpha= & 2 \pi \Lambda m_{D, \mathrm{eq}}^{2} \frac{\omega^{2}-q^{2}}{q^{2}} P^{00} \\
i \delta= & 2 \pi \Lambda m_{D, \mathrm{eq}}^{2} \frac{\omega / q}{\sqrt{q^{2}-(\mathbf{q} \cdot \mathbf{n})}}\left(P^{0 i} n^{i}-\frac{\mathbf{q} \cdot \mathbf{n} \omega}{q^{2}} P^{00}\right) \\
i \gamma= & 2 \pi \Lambda m_{D, \mathrm{eq}}^{2} \frac{1}{1-(\mathbf{q} \cdot \mathbf{n})^{2} / q^{2}} \\
& \times\left(P^{i j} n^{i} n^{j}-\frac{2 \mathbf{q} \cdot \mathbf{n} \omega}{q^{2}} P^{0 i} n^{i}+\frac{(\mathbf{q} \cdot \mathbf{n})^{2} \omega^{2}}{q^{4}} P^{00}\right)
\end{aligned}
$$

$$
i \beta=-i \alpha-i \gamma
$$

For the convenience of the reader we also collect the components of the retarded self-energy in Eq. (32):

$$
-i \Pi_{\mathrm{ret}}^{\mu \nu}=\Pi_{L} P_{L}^{\mu \nu}+\Pi_{e} E^{\mu \nu}+\Pi_{c} C^{\mu \nu}+\Pi_{d} D^{\mu \nu} .
$$

They were first derived in [43]. They have the same form as the components of $\Pi_{a a}$ except that $2 \pi \Lambda m_{D \text {, eq }}^{2}$ is replaced by $\frac{1}{2} m_{D, \text { eq }}^{2}$ and the angular integrals become

$$
\begin{aligned}
P_{\mathrm{ret}}^{00}= & \int_{-1}^{1} d z \frac{1}{\left(1+\xi z^{2}\right)^{2}}\left[-1+\left(\omega+\xi q_{\|} z\right)\right. \\
& \left.\times R\left(\omega-q_{\|} z, q_{\perp} \sqrt{1-z^{2}}\right)\right], \\
P_{\mathrm{ret}}^{0 i} n^{i}= & \int_{-1}^{1} d z \frac{z}{\left(1+\xi z^{2}\right)^{2}}\left[-1+\left(\omega+\xi q_{\|} z\right)\right. \\
& \left.\times R\left(\omega-q_{\|} z, q_{\perp} \sqrt{1-z^{2}}\right)\right], \\
P_{\mathrm{ret}}^{i j} n^{i} n^{j}= & \frac{1+\xi}{\xi^{3 / 2}}\left(\arctan \sqrt{\xi}-\frac{\sqrt{\xi}}{1+\xi}\right)+\int_{-1}^{1} d z \frac{z^{2}}{\left(1+\xi z^{2}\right)^{2}} \\
& \times\left[-1+\left(\omega+\xi q_{\|} z\right) R\left(\omega-q_{\|} z, q_{\perp} \sqrt{1-z^{2}}\right)\right]
\end{aligned}
$$

where

$$
\begin{aligned}
R(a, b) & =\int_{0}^{2 \pi} \frac{d \phi}{2 \pi} \frac{1}{a-b \cos \phi+i \epsilon} \\
& =\theta\left(a^{2}-b^{2}\right) \frac{\operatorname{sgn}(a)}{\sqrt{a^{2}-b^{2}}}-\theta\left(b^{2}-a^{2}\right) \frac{i}{\sqrt{b^{2}-a^{2}}} .
\end{aligned}
$$

Furthermore,

$$
\Pi_{e}=-\Pi_{L}-\Pi_{c}+\frac{\arctan \sqrt{\xi}}{\sqrt{\xi}} m_{D, \mathrm{eq}}^{2} .
$$

\section{APPENDIX D: ENERGY LOSS IN AN UNSTABLE MEDIUM}

In Eq. (45) we showed that a contour $\alpha$ that goes above all instability poles in the frequency domain is needed to transform the retarded correlator to the time domain [15]. Assuming the factorization in Eq. (46) this gives that

$$
\begin{aligned}
G_{\text {ret }}\left(t_{x}-t_{y}, \mathbf{k}\right)= & \int \frac{d p^{0}}{2 \pi} e^{-i p^{0}\left(t_{x}-t_{y}\right)} \widehat{G}_{\text {ret }}\left(p^{0}, \mathbf{p}\right) \\
& +\theta\left(t_{x}-t_{y}\right) \sum_{i} A_{i} e^{\gamma_{i}\left(t_{x}-t_{y}\right)}
\end{aligned}
$$

which guarantees that $G_{\text {ret }}\left(t_{x}, t_{y}\right)=0$ for $t_{x}<t_{y}$, i.e., that all propagation is causal and that instabilities give exponential growth. If we were to use an incorrect contour along the real line, the retarded correlator in the time domain would become

$$
\int \frac{d p^{0}}{2 \pi} e^{-i p^{0}\left(t_{x}-t_{y}\right)} \widehat{G}_{\mathrm{ret}}\left(p^{0}, \mathbf{p}\right)+\theta\left(t_{y}-t_{x}\right) \sum_{i} A_{i} e^{\gamma_{i}\left(t_{x}-t_{y}\right)} .
$$

This is nonvanishing for $t_{x}<t_{y}$ and thus does not describe causal evolution.

Earlier work on heavy-quark energy loss in an anisotropic plasma [37,38] implicitly used a contour along the real line, 
i.e., Eq. (D2). An easy way to see this is to note that their end result for energy loss was Eq. (10) which we derived using regular Fourier transforms and not the contour $\alpha$. Alternatively, the origin of the incorrect contour can be understood using the classical derivation in Eq. (11). This relied on Eq. (12) from linear response theory which describes how an electric field is induced by a current. The linear response relation comes from Fourier transforming the fundamental relation for the electric field:

$$
\begin{aligned}
E^{i}(X)= & \partial_{x^{0}} \int d^{4} Y D_{\mathrm{ret}}^{i j}(X-Y) J_{\mathrm{ext}}^{j}(Y) \\
= & \partial_{x^{0}} \int d^{4} Y \int \frac{d^{4} Q}{(2 \pi)^{4}} e^{-i(X-Y) Q} D_{\text {ret }}^{i j}(Q) \\
& \times \int \frac{d^{4} K}{(2 \pi)^{4}} e^{-i K Y} J_{\mathrm{ext}}^{j}(K) .
\end{aligned}
$$

Doing the $Y$ integral then gives a delta function $\delta^{(4)}(Q-K)$, leading to Eq. (12). However, in a system with an instability, things are not so simple. To preserve causality, we must use the contour $\alpha$ for the $q^{0}$ integral in Eq. (D3). Thus $q^{0}$ can be in the upper half complex plane. For an instability pole $q^{0}=$ $i \gamma$, the integral is

$$
\int_{-\infty}^{x^{0}} d y^{0} e^{-i\left(x^{0}-y^{0}\right) q^{0}}=\int_{-\infty}^{x^{0}} d y^{0} e^{\left(x^{0}-y^{0}\right) \gamma}=\infty
$$

which is divergent. The interpretation here is straightforward. Assuming a plasma that has existed for a long time compared to the rate of energy loss, the jet parton will have sourced unstable fields a long time ago that will have had time to grow to very large amplitudes leading to extremely large energy loss. Thus using the setup of $[37,38]$ should give infinite energy loss due to instabilities. It should be emphasized that the calculations of heavy quark energy loss in [37,38] correctly got the involved contribution of fluctuating soft modes and hard scattering.

The correct procedure in an unstable plasma is to use equations in the time domain like in the first line of Eq. (D3) and start the system at some finite time $t_{0}=0$. Then the integral in Eq. (D4) is

$$
\int_{0}^{x^{0}} d y^{0} e^{\left(x^{0}-y^{0}\right) \gamma}=\frac{e^{\gamma x^{0}}-1}{\gamma} .
$$

For later times $t_{x}$ the system will have evolved and the retarded correlator should be modified. This requires numerical calculations and has been performed in, e.g., [22,36]. Alternatively, if one is simply interested in fluctuating modes such as in this paper, one could subtract instability poles below a cutoff $\omega_{\text {cut }}$.

Other earlier calculations of probes in an anisotropic plasma do not suffer from this flaw. Calculation of photon emission through two-to-two scattering (see, e.g., [62]) only uses a resummed quark propagator which has no instability poles [63]. Furthermore, the heavy-quark potential [50] describes equal-time correlators and thus there is no room for instabilities to grow.

\section{APPENDIX E: CORRECTION TO [15]}

In our earlier paper [15], we showed that the $r r$ propagator in an unstable plasma is

$$
\begin{aligned}
G_{r r}\left(x^{0}, y^{0}\right) \approx & \int \frac{d k^{0}}{2 \pi} \widehat{G}_{\mathrm{ret}}\left(k^{0}\right) \Pi_{a a}\left(k^{0}\right) \widehat{G}_{\mathrm{adv}}\left(k^{0}\right) e^{-i k^{0}\left(x^{0}-y^{0}\right)}+\sum_{i} \int \frac{d k^{0}}{2 \pi} \frac{A_{i}}{k^{0}-i \gamma_{i}} \Pi_{a a}\left(k^{0}\right) \widehat{G}_{\mathrm{adv}}\left(k^{0}\right)\left(e^{-i k^{0} x^{0}}-e^{\gamma_{i} x^{0}}\right) e^{i k^{0} y^{0}} \\
& +\sum_{j} \int \frac{d k^{0}}{2 \pi} \widehat{G}_{\mathrm{ret}}\left(k^{0}\right) \Pi_{a a}\left(k^{0}\right) \frac{A_{j}^{*}}{k^{0}+i \gamma_{j}} e^{-i k^{0} x^{0}}\left(e^{i k^{0} y^{0}}-e^{\gamma_{j} y^{0}}\right) \\
& +\sum_{i, j} \int \frac{d k^{0}}{2 \pi} \frac{A_{i}}{k^{0}-i \gamma_{i}} \Pi_{a a}\left(k^{0}\right) \frac{A_{j}^{*}}{k^{0}+i \gamma_{j}}\left(e^{-i k^{0} x^{0}}-e^{\gamma_{i} x^{0}}\right)\left(e^{i k^{0} y^{0}}-e^{\gamma_{j} y^{0}}\right)
\end{aligned}
$$

assuming the factorization in Eq. (46). The first term describes fluctuating modes, the last term describes instability modes, and the middle terms are cross-terms between fluctuating and instability modes. Using a set of controlled approximations, Eq. (E1) can be shown to be equivalent to Eq. (47) [15].

In [15], we gave a heuristic discussion of Eq. (E1). This discussion, given in Eqs. (37)-(39) of [15], contained some wrong signs which we correct here. We emphasize that all results of [15], including Eq. (E1), remain unaffected.

The $r r$ propagator can be written as

$$
\begin{aligned}
G_{r r}\left(x^{0}, y^{0}, \mathbf{k}\right)= & \int d w^{0} \int d z^{0} G_{\mathrm{ret}}\left(x^{0}, w^{0} ; \mathbf{k}\right) \\
& \times \Pi_{a a}\left(w^{0}, z^{0} ; \mathbf{k}\right) G_{\mathrm{adv}}\left(z^{0}, y^{0} ; \mathbf{k}\right) .
\end{aligned}
$$

Omitting dependence on the three-momentum $\mathbf{k}$, this is

$$
\begin{aligned}
G_{r r}\left(x^{0}, y^{0}\right)= & \int d w^{0} \int d z^{0} \int \frac{d k^{0}}{2 \pi} G_{\text {ret }}\left(x^{0}, w^{0}\right) \\
& \times e^{-i k^{0}\left(w^{0}-z^{0}\right)} \Pi_{a a}\left(k^{0}\right) G_{\text {adv }}\left(z^{0}, y^{0}\right)
\end{aligned}
$$

where we Fourier transformed the self-energy $\Pi_{a a}$. This equation can easily be evaluated schematically. A mode in the retarded propagator with energy $E$ and decay rate $\Gamma$ is

$$
G_{\text {ret }}\left(x^{0}, w^{0}\right) \sim \theta\left(x^{0}-w^{0}\right) e^{-i E\left(x^{0}-w^{0}\right)-\Gamma\left(x^{0}-w^{0}\right)}
$$

in the time domain. In thermal equilibrium, or generally any system starting at time $t_{0}=-\infty$, this contributes

$$
\int_{-\infty}^{x^{0}} d w^{0} e^{-i k^{0} w^{0}} e^{-i(E-i \Gamma)\left(x^{0}-w^{0}\right)}=\frac{i e^{-i k^{0} x^{0}}}{k^{0}-E+i \Gamma}
$$


to the $r r$ correlator in Eq. (E3). However, in a system that is started at time $t_{0}=0$, the contribution of this mode is

$$
\begin{aligned}
& \int_{0}^{x^{0}} d w^{0} e^{-i k^{0} w^{0}} e^{-i(E-i \Gamma)\left(x^{0}-w^{0}\right)} \\
& =\frac{i}{k^{0}-E+i \Gamma}\left[e^{-i k^{0} x^{0}}-e^{-i(E-i \Gamma) x^{0}}\right] .
\end{aligned}
$$

Generally speaking, for a pole $b$ in the retarded propagator, we should have a contribution

$$
\frac{1}{k^{0}-b}\left(e^{-i k^{0} x^{0}}-e^{-i b x^{0}}\right)
$$

in Eq. (E1). However, controlled approximation allows us to drop terms $e^{-i b x^{0}}$ when $b \sim g \Lambda$ since those terms either decay rapidly or oscillate too fast to contribute to momentum broadening [15]. This explains the form of (E1): For instability poles $b=i \gamma$, we get a contribution

$$
\frac{1}{k^{0}-i \gamma}\left(e^{-i k^{0} x^{0}}-e^{\gamma x^{0}}\right)
$$

while for poles with $b \sim g \Lambda$, we simply get a contribution

$$
\frac{1}{k^{0}-b} e^{-i k^{0} x^{0}}
$$

[1] B. V. Jacak and B. Muller, The exploration of hot nuclear matter, Science 337, 310 (2012).

[2] P. Aurenche, F. Gelis, and H. Zaraket, A Simple sum rule for the thermal gluon spectral function and applications, J. High Energy Phys. 05, 043 (2002).

[3] S. Caron-Huot, $\mathrm{O}(\mathrm{g})$ plasma effects in jet quenching, Phys. Rev. D 79, 065039 (2009).

[4] M. Panero, K. Rummukainen, and A. Schäfer, Lattice Study of the Jet Quenching Parameter, Phys. Rev. Lett. 112, 162001 (2014).

[5] G. D. Moore, S. Schlichting, N. Schlusser, and I. Soudi, Non-perturbative determination of collisional broadening and medium induced radiation in QCD plasmas, J. High Energy Phys. 10 (2021) 059.

[6] S. Hauksson, S. Jeon, and C. Gale, Photon emission from quarkgluon plasma out of equilibrium, Phys. Rev. C 97, 014901 (2018).

[7] M. Strickland, J. Noronha, and G. S. Denicol, Anisotropic nonequilibrium hydrodynamic attractor, Phys. Rev. D 97, 036020 (2018).

[8] P. B. Arnold, G. D. Moore, and L. G. Yaffe, Effective kinetic theory for high temperature gauge theories, J. High Energy Phys. 01, 030 (2003).

[9] A. Kurkela, A. Mazeliauskas, J.-F. Paquet, S. Schlichting, and D. Teaney, Effective kinetic description of event-by-event pre-equilibrium dynamics in high-energy heavy-ion collisions, Phys. Rev. C 99, 034910 (2019).

[10] A. Kurkela, A. Mazeliauskas, J.-F. Paquet, S. Schlichting, and D. Teaney, Matching the Nonequilibrium Initial Stage of Heavy Ion Collisions to Hydrodynamics with QCD Kinetic Theory, Phys. Rev. Lett. 122, 122302 (2019).
[11] A. Kurkela and Y. Zhu, Isotropization and Hydrodynamization in Weakly Coupled Heavy-Ion Collisions, Phys. Rev. Lett. 115, 182301 (2015).

[12] M. C. Abraao York, A. Kurkela, E. Lu, and G. D. Moore, UV cascade in classical Yang-Mills theory via kinetic theory, Phys. Rev. D 89, 074036 (2014).

[13] P. B. Arnold, G. D. Moore, and L. G. Yaffe, Photon emission from ultrarelativistic plasmas, J. High Energy Phys. 11, 057 (2001).

[14] P. B. Arnold, G. D. Moore, and L. G. Yaffe, Photon emission from quark gluon plasma: Complete leading order results, J. High Energy Phys. 12, 009 (2001).

[15] S. Hauksson, S. Jeon, and C. Gale, Probes of the quark-gluon plasma and plasma instabilities, Phys. Rev. C 103, 064904 (2021).

[16] S. Mrowczynski, B. Schenke, and M. Strickland, Color instabilities in the quark-gluon plasma, Phys. Rep. 682, 1 (2017).

[17] J. Berges, K. Boguslavski, S. Schlichting, and R. Venugopalan, Turbulent thermalization process in heavy-ion collisions at ultrarelativistic energies, Phys. Rev. D 89, 074011 (2014).

[18] J. Berges, K. Boguslavski, S. Schlichting, and R. Venugopalan, Universal attractor in a highly occupied non-Abelian plasma, Phys. Rev. D 89, 114007 (2014).

[19] J. Berges, K. Boguslavski, S. Schlichting, and R. Venugopalan, Universality Far from Equilibrium: From Superfluid Bose Gases to Heavy-Ion Collisions, Phys. Rev. Lett. 114, 061601 (2015).

[20] K. Boguslavski, A. Kurkela, T. Lappi, and J. Peuron, Heavy quark diffusion in an overoccupied gluon plasma, J. High Energy Phys. 09, 077 (2020). 
[21] B. Schenke, M. Strickland, A. Dumitru, Y. Nara, and C. Greiner, Transverse momentum diffusion and jet energy loss in nonAbelian plasmas, Phys. Rev. C 79, 034903 (2009).

[22] S. Mrowczynski, Heavy Quarks in Turbulent QCD Plasmas, Eur. Phys. J. A 54, 43 (2018).

[23] A. Dumitru, Y. Nara, B. Schenke, and M. Strickland, Jet broadening in unstable non-Abelian plasmas, Phys. Rev. C 78, 024909 (2008).

[24] A. Ipp, D. I. Müller, and D. Schuh, Jet momentum broadening in the pre-equilibrium Glasma, Phys. Lett. B 810, 135810 (2020).

[25] A. Ipp, D. I. Müller, and D. Schuh, Anisotropic momentum broadening in the 2+1D Glasma: analytic weak field approximation and lattice simulations, Phys. Rev. D 102, 074001 (2020).

[26] M. E. Carrington, A. Czajka, and S. Mrowczynski, Heavy Quarks Embedded in Glasma, Nucl. Phys. A 1001, 121914 (2020).

[27] A. Kumar, A. Majumder, and J. H. Weber, Jet transport coefficient $\hat{q}$ in (2+1)-flavor lattice QCD, arXiv:2010.14463.

[28] A. Majumder, B. Muller, and S. Mrowczynski, Momentum Broadening of a Fast Parton in a Perturbative Quark-Gluon Plasma, Phys. Rev. D 80, 125020 (2009).

[29] M. E. Carrington, S. Mrówczyński, and B. Schenke, Momentum broadening in unstable quark-gluon plasma, Phys. Rev. C 95, 024906 (2017).

[30] A. V. Sadofyev, M. D. Sievert, and I. Vitev, Ab Initio Coupling of Jets to Collective Flow in the Opacity Expansion Approach, Phys. Rev. D 104, 094044 (2021).

[31] J. Ghiglieri and D. Teaney, Parton energy loss and momentum broadening at NLO in high temperature QCD plasmas, Int. J. Mod. Phys. E 24, 1530013 (2015).

[32] M. Le Bellac, Thermal Field Theory, Cambridge Monographs on Mathematical Physics (Cambridge University, New York, 2011).

[33] J. Ghiglieri, A. Kurkela, M. Strickland, and A. Vuorinen, Perturbative Thermal QCD: Formalism and Applications, Phys. Rep. 880, 1 (2020).

[34] S. Mrowczynski, Energy loss of a high-energy parton in the quark - gluon plasma, Phys. Lett. B 269, 383 (1991).

[35] M. H. Thoma and M. Gyulassy, Quark damping and energy loss in the high temperature QCD, Nucl. Phys. B 351, 491 (1991).

[36] M. E. Carrington, K. Deja, and S. Mrowczynski, Energy Loss in Unstable Quark-Gluon Plasma, Phys. Rev. C 92, 044914 (2015).

[37] P. Romatschke and M. Strickland, Energy loss of a heavy fermion in an anisotropic QED plasma, Phys. Rev. D 69, 065005 (2004).

[38] P. Romatschke and M. Strickland, Collisional energy loss of a heavy quark in an anisotropic quark-gluon plasma, Phys. Rev. D 71, 125008 (2005).

[39] E. Braaten and M. H. Thoma, Energy loss of a heavy fermion in a hot plasma, Phys. Rev. D 44, 1298 (1991).

[40] E. Braaten and M. H. Thoma, Energy loss of a heavy quark in the quark - gluon plasma, Phys. Rev. D 44, R2625 (1991).

[41] R. Baier and Y. Mehtar-Tani, Jet quenching and broadening: The Transport coefficient q-hat in an anisotropic plasma, Phys. Rev. C 78, 064906 (2008).
[42] P. Romatschke, Momentum broadening in an anisotropic plasma, Phys. Rev. C 75, 014901 (2007).

[43] P. Romatschke and M. Strickland, Collective modes of an anisotropic quark gluon plasma, Phys. Rev. D 68, 036004 (2003).

[44] M. E. Carrington, B. M. Forster, and S. Makar, Collective modes in anisotropic systems, Phys. Rev. C 104, 064908 (2021).

[45] B. S. Kasmaei and M. Strickland, Parton self-energies for general momentum-space anisotropy, Phys. Rev. D 97, 054022 (2018).

[46] J.-P. Blaizot and E. Iancu, The Quark gluon plasma: Collective dynamics and hard thermal loops, Phys. Rep. 359, 355 (2002).

[47] S. Mrowczynski and M. H. Thoma, Hard loop approach to anisotropic systems, Phys. Rev. D 62, 036011 (2000).

[48] M. E. Carrington, K. Deja, and S. Mrowczynski, Plasmons in Anisotropic Quark-Gluon Plasma, Phys. Rev. C 90, 034913 (2014).

[49] J. I. Kapusta and C. Gale, Finite-Temperature Field Theory: Principles and Applications, Cambridge Monographs on Mathematical Physics (Cambridge University, New York, 2011).

[50] A. Dumitru, Y. Guo, and M. Strickland, The Heavy-quark potential in an anisotropic (viscous) plasma, Phys. Lett. B 662, 37 (2008).

[51] M. Nopoush, Y. Guo, and M. Strickland, The static hard-loop gluon propagator to all orders in anisotropy, J. High Energy Phys. 09, 063 (2017).

[52] S. Mrowczynski, Stream Instabilities of the Quark-Gluon Plasma, Phys. Lett. B 214, 587 (1988); 656, 273(E) (2007).

[53] S. Mrowczynski, Plasma instability at the initial stage of ultrarelativistic heavy ion collisions, Phys. Lett. B 314, 118 (1993).

[54] P. B. Arnold, J. Lenaghan, and G. D. Moore, QCD plasma instabilities and bottom up thermalization, J. High Energy Phys. 08, 002 (2003).

[55] S. Hauksson, S. Jeon, and C. Gale, Hard probes of nonequilibrium quark-gluon plasma, Nucl. Phys. A 1005, 121888 (2021).

[56] A. Kurkela and G. D. Moore, Thermalization in Weakly Coupled Nonabelian Plasmas, J. High Energy Phys. 12, 044 (2011).

[57] P. Romatschke and M. Strickland, Collective modes of an anisotropic quark-gluon plasma II, Phys. Rev. D 70, 116006 (2004)

[58] A. Kurkela and U. A. Wiedemann, Analytic structure of nonhydrodynamic modes in kinetic theory, Eur. Phys. J. C 79, 776 (2019).

[59] P. B. Arnold and C. Dogan, QCD Splitting/Joining Functions at Finite Temperature in the Deep LPM Regime, Phys. Rev. D 78, 065008 (2008).

[60] P. B. Arnold, G. D. Moore, and L. G. Yaffe, Photon and gluon emission in relativistic plasmas, J. High Energy Phys. 06, 030 (2002).

[61] J. Casalderrey-Solana and D. Teaney, Transverse Momentum Broadening of a Fast Quark in a $\mathrm{N}=4$ Yang Mills Plasma, J. High Energy Phys. 04, 039 (2007).

[62] B. Schenke and M. Strickland, Photon production from an anisotropic quark-gluon plasma, Phys. Rev. D 76, 025023 (2007).

[63] B. Schenke and M. Strickland, Fermionic Collective Modes of an Anisotropic Quark-Gluon Plasma, Phys. Rev. D 74, 065004 (2006). 